\title{
Protective effect of HINT2 on mitochondrial function via repressing MCU complex activation attenuates cardiac microvascular ischemia-reperfusion injury
}

\author{
Su Li ${ }^{1}$ Jinxiang Chen ${ }^{1} \cdot$ Muyin $\mathrm{Liu}^{1} \cdot$ Yuqiong Chen ${ }^{3} \cdot$ Yuan $\mathrm{Wu}^{4} \cdot \mathrm{Qiyu} \mathrm{Li}^{1} \cdot$ Teng Ma $^{5}$. Jinfeng Gao ${ }^{1} \cdot$ Yan Xia ${ }^{1,2}$.

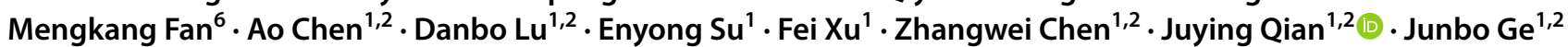

Received: 7 July 2021 / Revised: 17 November 2021 / Accepted: 18 November 2021 / Published online: 16 December 2021

(c) The Author(s) 2021

\begin{abstract}
Current evidence indicates that coronary microcirculation is a key target for protecting against cardiac ischemia-reperfusion $(\mathrm{I} / \mathrm{R})$ injury. Mitochondrial calcium uniporter $(\mathrm{MCU})$ complex activation and mitochondrial calcium $\left(\left[\mathrm{Ca}^{2+}\right]_{\mathrm{m}}\right)$ overload are underlying mechanisms involved in cardiovascular disease. Histidine triad nucleotide-binding 2 (HINT2) has been reported to modulate $\left[\mathrm{Ca}^{2+}\right]_{\mathrm{m}}$ via the MCU complex, and our previous work demonstrated that HINT2 improved cardiomyocyte survival and preserved heart function in mice with cardiac ischemia. This study aimed to explore the benefits of HINT2 on cardiac microcirculation in I/R injury with a focus on mitochondria, the MCU complex, and $\left[\mathrm{Ca}^{2+}\right]_{\mathrm{m}}$ overload in endothelial cells. The present work demonstrated that HINT2 overexpression significantly reduced the no-reflow area and improved microvascular perfusion in I/R-injured mouse hearts, potentially by promoting endothelial nitric oxide synthase (eNOS) expression and phosphorylation. Microvascular barrier function was compromised by reperfusion injury, but was repaired by HINT2 overexpression via inhibiting VE-Cadherin phosphorylation at $\mathrm{Tyr}^{731}$ and enhancing the VE-Cadherin/ $\beta$-Catenin interaction. In addition, HINT2 overexpression inhibited the inflammatory response by suppressing vascular cell adhesion molecule-1 (VCAM-1) and intercellular adhesion molecule-1 (ICAM-1). Mitochondrial fission occurred in cardiac microvascular endothelial cells (CMECs) subjected to oxygen-glucose deprivation/reoxygenation (OGD/R) injury and resulted in mitochondrial dysfunction and mitochondrion-dependent apoptosis, the effects of which were largely relieved by HINT2 overexpression. Additional experiments confirmed that $\left[\mathrm{Ca}^{2+}\right]_{\mathrm{m}}$ overload was an initiating factor for mitochondrial fission and that HINT2 suppressed $\left[\mathrm{Ca}^{2+}\right]_{\mathrm{m}}$ overload via modulation of the MCU complex through directly interacting with MCU in CMECs. Regaining $\left[\mathrm{Ca}^{2+}\right]_{\mathrm{m}}$ overload by spermine, an MCU agonist, abolished all the protective effects of HINT2 on OGD/R-injured CMECs and I/R-injured cardiac microcirculation. In conclusion, the present report demonstrated that HINT2 overexpression inhibited MCU complex-mitochondrial calcium overload-mitochondrial fission and apoptosis pathway, and thereby attenuated cardiac microvascular ischemia-reperfusion injury.
\end{abstract}

Keywords Cardiac ischemia-reperfusion injury $\cdot$ Cardiac microvascular injury $\cdot$ HINT2 $\cdot$ MCU complex $\cdot$ Mitochondrial calcium overload $\cdot$ Mitochondrial fission

Su Li, Jinxiang Chen, and Muyin Liu have contributed equally to this work.

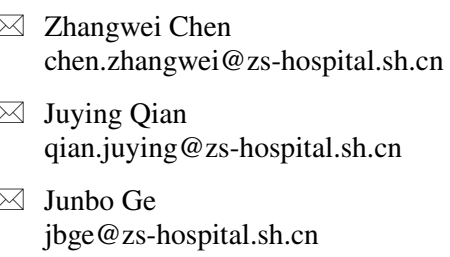

Extended author information available on the last page of the article

\section{Introduction}

The reperfusion strategy is the most widely acknowledged treatment for acute myocardial infarction (MI) [22]. However, resupply blood with ischemic myocardium causes ischemia-reperfusion (I/R) injury, which is a clinical reality and a problem that needs much more attention [22]. In recent years, the importance of microcirculation in the protection of cardiac function has become more apparent [18, 19]. The mechanisms of I/R injury to coronary microvascular impairment range from microembolization, impaired 
vasomotion, capillary plugging and rupture, to leukocyte infiltration, which are partly different from those contributing to cardiomyocyte injury $[18,19]$. Moreover, these pathological changes further progress to cardiac edema and even coronary no-reflow phenomena, thereby discounting the benefits of reperfusion therapy [18]. Considering that coronary microvascular dysfunction has been recognized as a determinant for patients' adverse outcomes, it is highly necessary to better understand the underlying mechanisms and search for effective therapeutic targets [8, 22].

Mitochondria are dynamic organelles that perform selfrepair via mitochondrial fission and fusion, two distinctly opposite processes in which one separates the damaged fractions from the whole mitochondrial body and the other merges two fractions into a healthy mitochondrion [39]. The balance of fission and fusion is critical to maintain mitochondrial and endothelial function [7, 28]. However, mitochondrial fission in the coronary microcirculation is excessively activated after reperfusion, which further accentuates mitochondrial reactive oxygen species (ROS) accumulation, mitochondrial permeability transition pore (mPTP) opening, and apoptosis activation, ultimately resulting in microvascular dysfunction [51]. Additionally, reperfusion injuryelicited mitochondrial dysfunction has been implicated in ROS bursts, inflammatory response activation, endothelial swelling, leukocyte aggregates, and altered vasomotion, all of which inevitably contribute to microthrombus formation and microvascular obstruction [13]. In contrast, treatments aimed at mitochondrial repair and ROS elimination alleviated microvascular dysfunction under I/R injury and showed the potential to relieve the no-reflow phenomenon [48]. The above evidence strongly supports the targeting of mitochondrial fission and function as a promising approach for cardiac and microvascular protection from I/R injury.

Calcium overload has been identified as an early sign of apoptosis in cardiomyocytes after I/R injury [41]. Persistent calcium oscillation triggers mitochondrial dysfunction, leads to oxidative stress and mPTP opening, and ultimately activates mitochondrion-dependent apoptosis [36]. This death pattern contributes to many types of cardiovascular diseases, including cardiac I/R injury and heart failure [10, 25]. However, the pathological role of calcium overload in microvascular reperfusion injury is a neglected target for cardioprotection. Limited evidence has proven that the restriction of endothelial mitochondrial calcium $\left(\left[\mathrm{Ca}^{2+}\right]_{m}\right)$ influx exerted benefits on cardiac microcirculation, as evidenced by enhanced microvascular perfusion, reduced microvascular obstruction, inhibited inflammatory cell infiltration, and decreased infarction size in a mouse cardiac I/R model, and the potentially involved mechanisms were largely attributed to mitigated mitochondrial morphological and functional damage $[27,47] .\left[\mathrm{Ca}^{2+}\right]_{\mathrm{m}}$ influx is primarily mediated by the mitochondrial calcium uniporter (MCU) complex, which mainly consists of four MCU subunits and essential MCU regulators (EMREs) [2, 46]. The regulatory proteins that interact with MCU subunits include mitochondrial calcium uptake 1/2 (MICU1/2) and MCU dominant-negative $\beta$-subunit (MCUb) $[2,46]$. As its core component, the four MCU subunits were mainly in charge of mitochondrial calcium uptake and were upregulated by $\mathrm{I} / \mathrm{R}$ injury, resulting in $\left[\mathrm{Ca}^{2+}\right]_{\mathrm{m}}$ overload and mitochondrial dysfunction [49]. In contrast, the inhibition of MCU alleviated cardiomyocyte apoptosis and myocardial reperfusion injury [15, 49]. The regulation and contribution of MCU activity was also highlighted in endothelial cells, and has been proven to be involved in ischemic microvascular diseases and atherosclerotic vascular disease [1]. Previous work suggested that the inhibition of MCU in the endothelium restrained $\left[\mathrm{Ca}^{2+}\right]_{\mathrm{m}}$ uptake and thereby improved coronary microvascular function in the face of I/R injury [27]. However, the detailed mechanisms by which the MCU complex regulates mitochondrial function and microvascular protection have not been fully explored.

Histidine triad (HIT) nucleotide-binding protein 2 (HINT2) is located in mitochondria and belongs to the HINT branch of the HIT superfamily [5]. Genetic ablation of HINT2 in mice resulted in oxidative respiratory chain impairment and highly acetylated mitochondrial proteins, which resembled the pattern of mitochondrial injury after hypoxia [33]. Our previous work verified that HINT2 overexpression preserved mouse heart function after MI, and maintained mitochondrial membrane potential (MMP) and mitochondrial respiration in cardiomyocytes exposed to hypoxia [11]. In addition, there is evidence that HINT2 can modulate intracellular calcium $\left(\left[\mathrm{Ca}^{2+}\right]_{\mathrm{i}}\right)$ and $\left[\mathrm{Ca}^{2+}\right]_{\mathrm{m}}$ dynamics in hepatocytes and enhance apoptosis in pancreatic cancer cells by regulating the MCU complex [6,35]. However, the exact role of HINT2 in I/R-induced cardiac microvascular injury has not been reported, and whether HINT2 can govern $\left[\mathrm{Ca}^{2+}\right]_{\mathrm{m}}$ fluctuation via the MCU complex and thereby improve mitochondrial dynamics and function in endothelial cells remains unknown. Therefore, the present study was designed to evaluate the effect of HINT2 overexpression on coronary microvascular protection in the setting of cardiac I/R injury and to explore the possible mechanisms with a focus on mitochondrial dynamics and MCU complexrelated $\left[\mathrm{Ca}^{2+}\right]_{\mathrm{m}}$ homeostasis.

\section{Methods}

\section{Endothelial HINT2 overexpression strategies}

Mouse HINT2-overexpressing adeno-associated virus-9 (AAV9-HINT2) genome particles containing the TIE promoter and Flag were obtained from Genomeditech (China, 
Shanghai). To establish an endothelial cell (EC)-specific HINT2-overexpressing mouse model, $100 \mu \mathrm{l}$ AAV9-HINT2 particles or negative control (AAV9-NC) at a density of $5 \times 10^{\wedge} 12$ v.g./ml was injected into 6-week-old C57BL/6J mice via the caudal vein. Four weeks after transfection, cardiac tissues were sectioned to measure transfection efficiency of AAV9 in the cardiac microcirculation via co-immunofluorescence staining of Flag, cTnT, and CD31. Cardiac microvascular endothelial cells (CMECs) were isolated to measure HINT2 overexpression efficiency by Western blot.

Adenoviruses (ADVs) containing the HINT2 gene (ADVHINT2) and a negative control (ADV-NC) were obtained from Hanbio (China, Shanghai). To establish HINT2-overexpressing CMECs in vitro, CMECs were isolated from 6to 8-week-old C57BL/6J mice and transfected with ADVHINT2 or ADV-NC at different multiplicities of infection (MOIs). HINT2 overexpression efficiency was determined using Western blot. Plasmids containing the full-length HINT2, the HINT2 mutant and the negative control (NC) were obtained from Genomeditech (China, Shanghai) and transfected into CMECs with Lipofectamine 3000 (Thermo Fisher, USA).

\section{Cardiac I/R injury model}

Four weeks after AAV9-HINT2 transfection, male C57BL/6J mice (10 weeks of age) underwent surgical cardiac I/R injury as described previously [12]. The left anterior descending coronary artery (LAD) was ligated via a slipknot using 6-0 silk for $45 \mathrm{~min}$, and then, the slipknot was gently loosened to induce reperfusion injury. Mice in the sham group underwent the same procedures without tying the slipknot. To inhibit the MCU complex, $50 \mathrm{nmol} / \mathrm{kg}$ Ru360 (MCE, USA) was injected intraperitoneally into AAV9-NC mice $1 \mathrm{~h}$ before I/R injury [15]. Spermine (5 mg/kg, MCE, USA) was injected intraperitoneally into AAV9-HINT2 mice $1 \mathrm{~h}$ before I/R injury to activate the MCU complex [27].

\section{CMECs isolation, oxygen-glucose deprivation/ reoxygenation (OGD/R) injury, and cell viability assays}

CMECs were isolated from the left ventricle according to a previous method [24]. Briefly, after the left ventricle were sufficiently digested into single cell suspension with liberase (Roche, Switzerland), CMECs were gathered using CD31coupled microbeads (Thermo Fisher, USA) and further cultured in endothelial culture medium (ECM, ScienCell, USA) containing endothelial cell growth supplements (ECGS), 5\% fetal bovine serum (FBS), $100 \mathrm{U} / \mathrm{ml}$ penicillin, and $100 \mu \mathrm{g} /$ $\mathrm{ml}$ streptomycin. The purity of isolated CMECs was identified by immunofluorescence staining of CD31.
To induce OGD/R injury, CMECs were cultured in Eagle's solution (Genom, China) and maintained in a hypoxia chamber (MGC, Japan) with a gas mixture of 5\% $\mathrm{CO}_{2}$ and $95 \% \mathrm{~N}_{2}$ at $37{ }^{\circ} \mathrm{C}$ for $6 \mathrm{~h}$ to induce OGD injury. Reoxygenation injury was triggered by restoring the culture medium and atmosphere $\left(5 \% \mathrm{CO}_{2}\right.$ and $95 \%$ air). Cells transfected with ADV-NC were pretreated with $10 \mu \mathrm{M}$ Ru360 $1 \mathrm{~h}$ before OGD/R injury to inhibit the MCU complex. Mdivi-1 (5 $\mu \mathrm{M}, \mathrm{MCE}$, USA) was added to the ADV-NC group during OGD/R injury to inhibit mitochondrial fission. In contrast, $1 \mu \mathrm{M}$ FCCP (MCE, USA) or $10 \mu \mathrm{M}$ spermine was applied to ADV-HINT2 cells for $2 \mathrm{~h}$ to reintroduce mitochondrial fission or reactivate the MCU complex, respectively. Cell viability was detected by a Cell Counting Kit- 8 assay kit (EpiZymi, China).

\section{Thioflavin S staining assays}

Thioflavin S staining assay was used to measure the myocardial no-reflow phenomenon $[30,44]$. Briefly, $6 \%$ thioflavin $\mathrm{S}$ (1 $\mathrm{ml} / \mathrm{kg}, \mathrm{MCE}, \mathrm{USA})$ was injected into mice via the tail vein and allowed to circulate for 1-2 min. The hearts were quickly harvested, fixed in $4 \%$ paraformaldehyde (PFA) overnight, cut into $1 \mathrm{~mm}$ sections, and then observed under a stereomicroscope (Leica, Germany). $359 \mathrm{~nm}$ excitation light was used for imaging. The reflow area showed bright blue fluorescence, whereas the no-reflow area was dark. The no-reflow degree was indicated as the percentage of dark area to the left ventricle.

\section{Triphenyltetrazolium chloride (TTC) staining}

TTC staining was conducted to measure cardiac infarction size [4]. After the mice were anesthetized with $2 \%$ isoflurane, the slipknot was tied again, and $1 \%$ Evans blue (w/v, Sigma, USA) was injected into the aortic root to perfuse the left ventricle. Then, the heart was rapidly excised, cut into $1 \mathrm{~mm}$ slices, and incubated with 1\% TTC solution (Sigma, USA) at $37{ }^{\circ} \mathrm{C}$ for $10 \mathrm{~min}$. Consecutive slices in each sample were scanned by a white light scanner (Canon, Japan). Area at risk (AAR) was defined as tissues not perfused by Evans blue. Infarcted myocardium was white, whereas viable myocardium was red. The infarction degree was calculated as the ratio of the infarction area to the AAR.

\section{Modified Miles assays}

Evans blue (1\%, w/v, $100 \mu \mathrm{l})$ was injected into mice via the tail vein. The mice were sacrificed $30 \mathrm{~min}$ after injection, and the hearts were perfused with citrate buffer $(\mathrm{pH} 4.5)$ through the aorta to evacuate blood. Left ventricle samples were immersed in $1 \mathrm{ml}$ formamide, homogenized, and incubated at $60{ }^{\circ} \mathrm{C}$ overnight to elute Evans blue. The samples 
were centrifuged at $10,000 \times g$ for 40 min to extract the supernatant containing Evans blue. Absorbance at $620 \mathrm{~nm}$ was read by a FlexStation 3 instrument (Molecular Devices, USA). The concentration of Evans blue was calculated from a standard curve and corrected by left ventricle weight.

\section{Immunofluorescence staining}

Frozen sections $(6 \mu \mathrm{m})$ were fixed with cold acetone for $10 \mathrm{~min}$ at $-20{ }^{\circ} \mathrm{C}$, air dried for $15 \mathrm{~min}$, and blocked with $5 \%$ bovine serum albumin (BSA) for $1 \mathrm{~h}$ at room temperature (RT). The sections were then incubated with primary antibodies. After overnight incubation at $4{ }^{\circ} \mathrm{C}$, the samples were washed with PBS and incubated with fluorescence-labeled secondary antibodies for $1 \mathrm{~h}$ at RT. To measure microvascular perfusion, $100 \mu \mathrm{l}$ FITC-combined tomato lectin $(1 \mathrm{mg} /$ $\mathrm{ml}$, Sigma, USA) was injected into mice via the tail vein and allowed to circulate for $10 \mathrm{~min}$ [31, 42]. Then, heart samples were harvested, sectioned, and stained for CD31 to label vessels. The microvascular perfusion ratio was indicated as the percentage of lectin-positive microvessels to CD31-positive microvessels. Immunofluorescence staining of cell samples was performed with the same procedures, except the samples were fixed with 4\% PFA for $10 \mathrm{~min}$ and permeabilized with $0.5 \%$ Triton X-100 for $10 \mathrm{~min}$. Detailed information of the primary and secondary antibodies used in immunofluorescence staining, including source, catalog number, and dilution, is listed in Table S1. All tissue sections and confocal dishes were observed under a confocal microscope (Olympus FV3000, Japan). At least five random fields were captured and quantitatively analyzed via ImageJ (version $1.53 \mathrm{c}, \mathrm{NIH}, \mathrm{USA}$ ).

\section{Cell fluorescence staining}

Mitochondria were stained with MitoTracker ${ }^{\circledR}$ Deep Red FM (200 nM, Invitrogen ${ }^{\mathrm{TM}}$, USA) for $30 \mathrm{~min}$ in the dark. Cellular ROS and mitochondrial ROS (mtROS) were measured by CellROX ${ }^{\circledR}$ Deep Red Reagent $\left(5 \mu \mathrm{M}\right.$, Invitrogen ${ }^{\mathrm{TM}}$, USA) and MitoSOX ${ }^{\mathrm{TM}}$ Red Mitochondrial Superoxide Indicator ( $5 \mu \mathrm{M}$, Invitrogen ${ }^{\mathrm{TM}}$, USA) for $30 \mathrm{~min}$, respectively. To determine mitochondrial membrane potential (MMP), the cells were incubated with $2 \mu \mathrm{M} \mathrm{JC}-1$ probe (Beyotime, China) for $30 \mathrm{~min}$. MMP was calculated as the ratio of redto-green fluorescence intensity according to the instructions. Cells were fixed, permeabilized, and then incubated with TUNEL (Sigma-Aldrich, USA) reaction mixture buffer for $60 \mathrm{~min}$ to detect apoptosis. $\left[\mathrm{Ca}^{2+}\right]_{\mathrm{i}}$ and $\left[\mathrm{Ca}^{2+}\right]_{\mathrm{m}}$ levels were measured with $5 \mu \mathrm{M}$ Fluo- 4 and $1 \mu \mathrm{M}$ Rhod-2 (Thermo Fisher, USA), respectively, for $30 \mathrm{~min}$ at $37^{\circ} \mathrm{C}$. Nuclei were labeled by DAPI (Beyotime, China). All the above fluorescence images were captured with a laser confocal microscope (Olympus FV3000, Japan). Fluorescence intensity was analyzed with ImageJ (Version $1.53 \mathrm{c}, \mathrm{NIH}, \mathrm{USA})$. Mitochondrial length was measured by MiNA (https://github. com/ScienceToolkit/MiNA), a plug-in in ImageJ.

\section{Nitrite detection}

The nitrite content in cardiac tissue and cell culture medium was measured by a Micro NO Content assay kit (Solarbio, China) according to the principle of detection. Cardiac nitrite content was standardized to the protein concentration of smashed cardiac tissue.

\section{Migration, wound healing, and capillary tube formation assays}

Migration assays were performed using Corning Transwell chambers ( $8 \mu \mathrm{m}$ pores, $6.5 \mathrm{~mm}$ diameter, USA) that were precoated with $10 \mu \mathrm{g} / \mathrm{ml}$ fibronectin (ScienCell, USA). After OGD/R injury, cells were digested and seeded in the upper chambers containing FBS-free ECM. ECM containing $10 \%$ FBS was then added to the lower chambers to stimulate migration. After $24 \mathrm{~h}$ of culture, cells that traversed through the membrane were fixed with 4\% PFA for $30 \mathrm{~min}$ and stained with $0.05 \%$ crystal violet solution (Solarbio, China) for $20 \mathrm{~min}$. A wound-healing assay was performed by scratching the cell monolayer with a $200 \mu$ pipette tip in 6-well plates. Cells were washed with PBS twice and cultured in ECM without FBS. Images were collected at the beginning and $48 \mathrm{~h}$ after scratching. The wound-healing speed was calculated as the ratio of the healed areas at $48 \mathrm{~h}$ to the original injured areas. A capillary tube formation assay was performed using the following procedures. Matrigel (50 $\mu \mathrm{l}$, BD Bioscience) was added to each well of a 96-well plate and solidified at $37^{\circ} \mathrm{C}$ for $30 \mathrm{~min}$. CMECs were digested and resuspended in ECM. A total of $5 \times 10^{4}$ cells were seeded in each well and cultured at $37^{\circ} \mathrm{C}$ for $3 \mathrm{~h}$. Capillary-like tubes were imaged by an optical microscope (Leica DM3000, Germany), and the numbers of branch points were counted manually.

\section{Endothelial monolayer permeability assays}

CMECs $\left(1 \times 10^{5}\right)$ were seeded in the upper chamber of a Corning Transwell $(0.4 \mu \mathrm{m}$ pore size, $6.5 \mathrm{~mm}$ diameter, USA) that was precoated with fibronectin. After OGD/R injury, $100 \mu$ FITC-BSA (68 kDa, Sigma, USA) was added to the upper chamber at a concentration of $1 \mathrm{mg} / \mathrm{ml}$. After $1 \mathrm{~h}$ of free permeation, the fluorescence intensity of FITCBSA in the upper and lower chambers was quantified by a FlexStation 3 (Molecular Devices, USA). 


\section{Protein sample preparation and Western blotting assay}

Total proteins were extracted in RIPA lysis buffer (Beyotime, China) on ice, followed by centrifugation at $12,000 \mathrm{rpm}$ for $30 \mathrm{~min}$ to remove cell fragments. Mitochondria were extracted by lysing cells with Mitochondrial Separation Reagent (Beyotime, China) on ice for $15 \mathrm{~min}$. After centrifugation, mitochondria were separated from cytoplasmic fragments and further lysed by Mitochondrial Lysis Buffer to extract mitochondrial protein. For immunoprecipitation (IP), cells were lysed with Cell Lysis Buffer for IP (Weiao Biological Company, China) on ice for $15 \mathrm{~min}$ and incubated with protein A/G-magnetic beads conjugated with antibodies against VE-Cadherin (Abcam, ab205336) and Flag (Abcam, ab205606) for $30 \mathrm{~min}$ at RT under constant rotation. Rabbit IgG (Abcam, ab172730) was used as a technical negative control to assess nonspecific binding. The immunocomplexes were mixed with loading buffer and boiled for $10 \mathrm{~min}$ to elute the target protein. Nondenatured protein samples were obtained by lysing cells with Native Lysis Buffer and mixing with NativePAGE loading buffer (Solarbio, China) without heating. Carbonylated mitochondrial proteins were detected by a protein oxidation detection kit (Sigma, USA). After extraction, mitochondrial proteins were incubated with 2.4-dinitrophenylhydrazine (DNPH) to conjugate carbonyl groups.

After quantified, protein samples (10-20 $\mu \mathrm{g}$ ) were loaded on SDS-polyacrylamide gels for electrophoresis and then transferred to PVDF membranes. After blocking with $5 \% \mathrm{BSA}$ for $1 \mathrm{~h}$, the membranes were incubated with primary antibodies overnight at $4{ }^{\circ} \mathrm{C}$. Later, after rinsing with TBST for three times, the membranes were incubated with horseradish peroxidase (HRP)-conjugated secondary antibodies (Weiao Biological Company, China) at RT for $1 \mathrm{~h}$. The blots were shown by Pierce ${ }^{\mathrm{TM}}$ enhanced chemiluminescence (ECL) Western blotting Substrate (Thermo Fisher, USA). The band intensities were analyzed by ImageJ (Version 1.53c, NIH, USA). Native-PAGE was performed for the detection of DRP1 oligomers according to the same procedure except nondenatured protein samples $(10 \mu \mathrm{g})$ were loaded on polyacrylamide gels without denaturants (EpiZyme, China). Detailed information of the primary antibodies used in immunoblotting, including source, catalog number, and dilution, is listed in Table S2.

\section{Structure-based protein interaction interface analysis}

The protein structures were predicted by Modeller (salilab. org/modeller) using Protein Data Bank (PDB) structure 6YI0 as template for HINT2 and 6058 as template for
MCU. Structures of proteins were submitted to the zdock tool (http://zdock.umassmed.edu/) to predict potential amino acid (AA) binding sites. Binding free energy (BFE) between proteins was calculated by MMGBSA analysis.

\section{Statistical analysis}

The data are expressed as the means \pm SEMs. The Shapiro-Wilk test and Kolmogorov-Smirnov test were performed before parametric tests, and the results showed that all obtained data were normally distributed. Statistical analyses were performed with Student's $t$ test or one-way analysis of variance (ANOVA) followed by post hoc tests. $P<0.05$ was considered statistically significant.

\section{Results}

\section{Endothelial-specific overexpression of HINT2 alleviated cardiac microvascular dysfunction after I/R injury}

In our mouse model of cardiac I/R injury, HINT2 was significantly reduced in both cardiac tissues and primary CMECs in ischemic region after $45 \mathrm{~min}$ of ischemia and reached the lowest point after $6 \mathrm{~h}$ of reperfusion (Fig. 1a, b). Therefore, mice suffering $6 \mathrm{~h}$ of reperfusion injury were selected as our following study subjects. To gain more insight into the role of HINT2 in microvascular protection, an AAV9mediated endothelial-specific HINT2 overexpression mouse model was established. Fluorescence staining of Flag indicated the transfection efficiency of AAV9 was $>95 \%$ in the microcirculation (Fig. S1a), and HINT2 signal was higher in the Flag-positive area in AAV9-HINT2-infected hearts (Fig. $\mathrm{S} 1 \mathrm{~b})$. Meanwhile, HINT2 expression in isolated CMECs was $~ 2.82$-fold higher than that in mice transfected with AAV9-NC (Fig. S1c). Moreover, HINT2 was not transfected into cardiomyocytes, as no obvious colocalization between Flag and cTnT was observed (Fig. S1a), and the expression of HINT2 was equal in cardiomyocytes isolated from the AAV9-NC and AAV9-HINT2 groups (Fig. S1d).

To examine the impact of HINT2 on microvascular damage, the no-reflow phenomenon was measured. An obvious perfusion defect of thioflavin $\mathrm{S}$ was observed in the left ventricle after reperfusion, and HINT2 overexpression significantly reduced the area of no-reflow (Fig. 1c, d). The fundamental pathology of the no-reflow phenomenon was highly related to compromised microvascular perfusion. In our mouse model, the microvasculature became sparse after reperfusion and was accompanied by a lower ratio of lectin-positive microvessels, suggesting decreased microvascular perfusion (Fig. 1e). In contrast, HINT2 overexpression obviously relieved perfusion defects (Fig. 1e). 


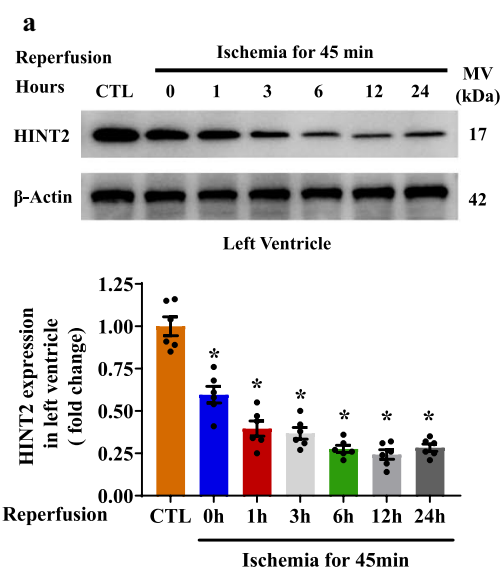

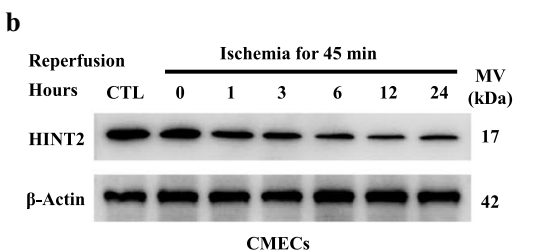

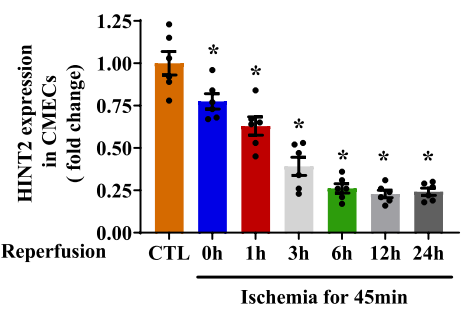

c
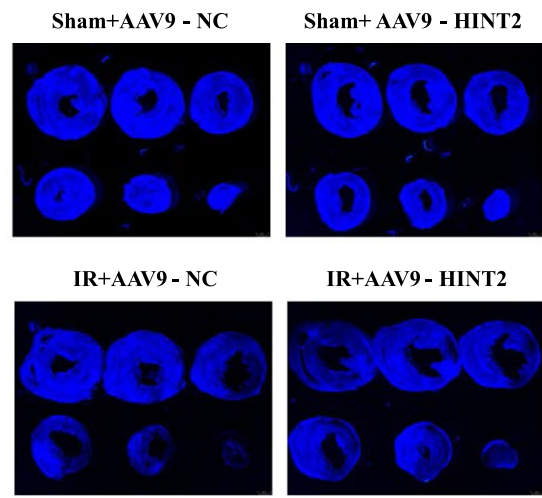

IR+AAV9 - HINT2

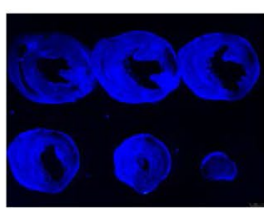

d

e

Sham
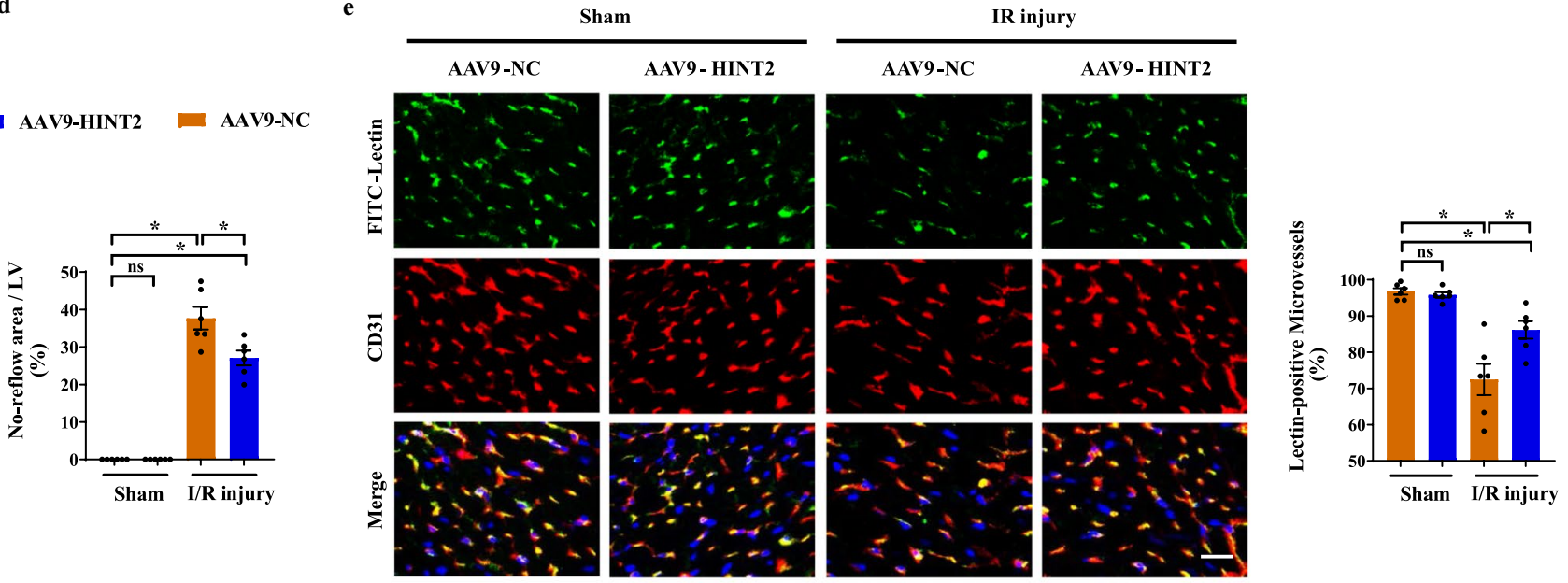

AAV9 - HINT2
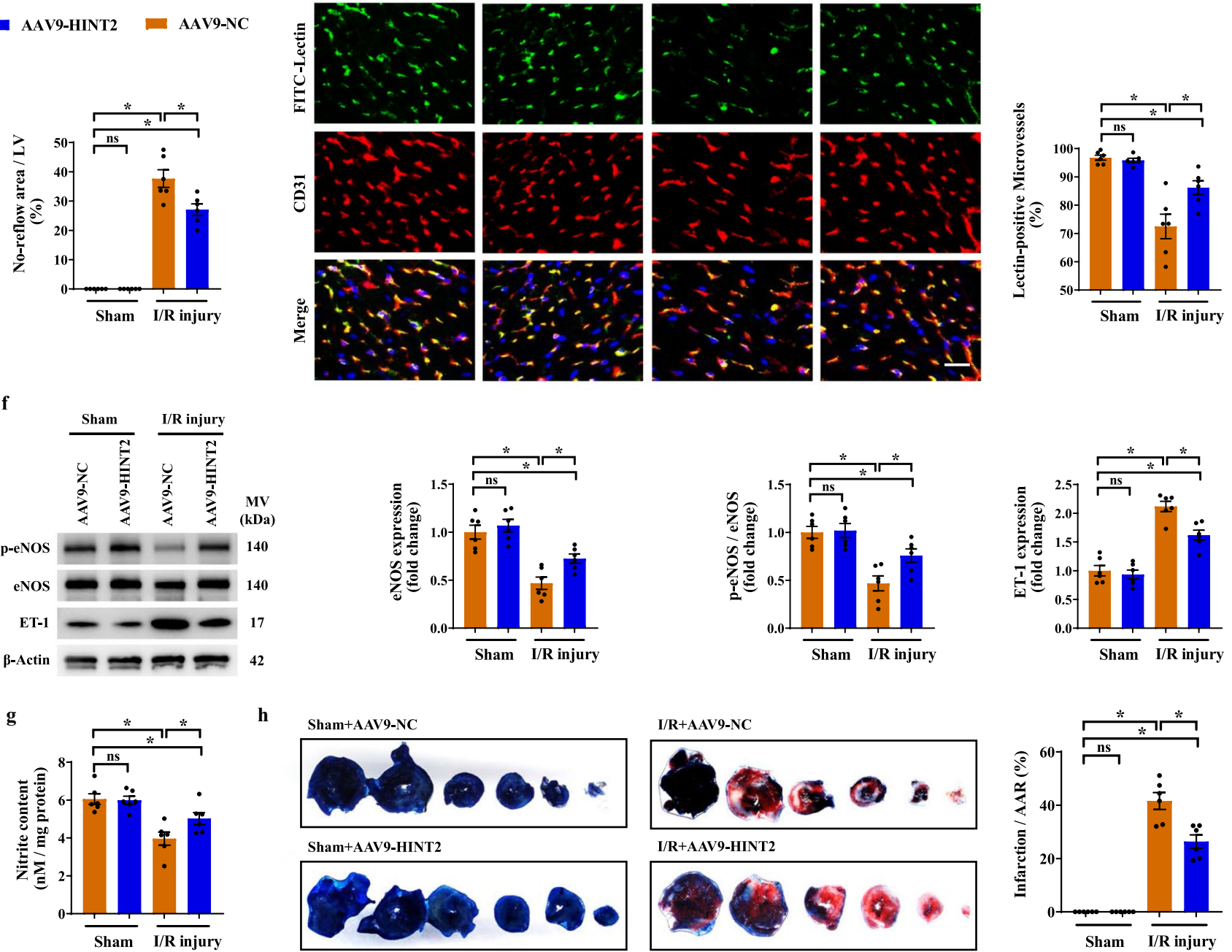

h
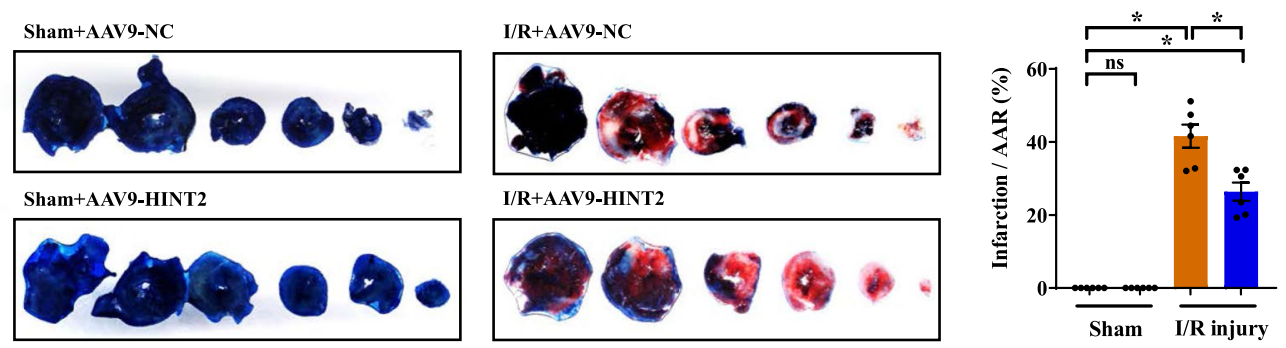

Microvascular patency is highly dependent on endotheliumcontrolled vasodilatation. Nitrite content, eNOS expression, and eNOS phosphorylation at $\mathrm{Ser}^{1177}$, a biologically active site, were significantly downregulated in ischemic ventricles after I/R injury, and the vascular constriction factor ET-1 was markedly increased (Fig. 1f, g). The above effects were obviously alleviated by HINT2 transfection (Fig. 1f, g). Further works revealed that I/R injury led to microvascular barrier collapse, as evidenced by higher Evans blue leakage in ischemic tissues (Fig. S1e). However, HINT2 overexpression 
४Fig. 1 Endothelial-specific overexpression of HINT2 alleviated cardiac microvascular injury and myocardial infarction after I/R injury. After $45 \mathrm{~min}$ of ischemia and 0-24 h of reperfusion, the expression of HINT2 in left ventricle and isolated CMECs was measured by Western blot analysis $(\mathbf{a}, \mathbf{b})$. $\mathbf{c}-\mathbf{h}$ Mice were transfected with AAV9-NC or AAV9-HINT2 and suffered from $45 \mathrm{~min}$ ischemia injury and $6 \mathrm{~h}$ reperfusion injury. No reflow area was detected by thioflavin $\mathrm{S}$ staining and quantified (c, d). Cardiac microvascular perfusion was indicated by the ratio of lectin-positive microvessels (green) to CD31-positive microvessels (red). Scale bars: $15 \mu \mathrm{m}$ (e). eNOS expression, eNOS phosphorylation at $\mathrm{Ser}^{1177}$, and ET-1 expression in left ventricle were detected by Western blot analysis (f). Nitrite content in left ventricle (g). Infarcted area was detected by TTC staining and quantified (h). ${ }^{*} P<0.05$, significantly different as indicated. $n s$ Not significant. Biological replicates were performed for six times in the above studies

significantly inhibited Evans blue permeation (Fig. S1e). Besides, VE-Cadherin were reduced in I/R injury, while a possible improvement of VE-Cadherin with HINT2 overexpression was observed, although the presented quantification still suggested a significant reduction compared to sham animals (Fig. S1f). Moreover, HINT2 overexpression reduced VE-Cadherin phosphorylation at $\mathrm{Tyr}^{731}$, a binding site for $\beta$-Catenin, and, correspondingly, enhanced the interaction between VE-Cadherin and $\beta$-Catenin during I/R injury (Fig. S1f, g). Ultimately, endothelial-specific overexpression of HINT2 reduced the infarction size (Fig. 1h), indicating that HINT2-mediated microvascular improvement inhibited myocardial necrosis in the acute reperfusion phase.

\section{HINT2 overexpression balanced mitochondrial dynamics in CMECs both in vivo and in vitro}

To elucidate the potential mechanism by which HINT2 protected the cardiac microvasculature against reperfusion injury, the present work focused on mitochondrial dynamics, an important perspective in cardiac microvascular diseases [7, 9]. Mitochondrial dynamics-related molecules were monitored in CMECs isolated from I/R models. After reperfusion injury, DRP1 and Fis1 were obviously upregulated in isolated CMECs, whereas MFN1 and MFN2 was notably reduced, indicating that mitochondrial fission may be enhanced (Fig. S2a, b). In comparison, the above molecular changes were reversed by HINT2 overexpression (Fig. S2a, b). To further clarify the mechanisms underlying HINT2-mediated mitochondrial dynamics, in vitro studies were performed by challenging CMECs with OGD/R injury. HINT2 expression gradually decreased during the reoxygenation stage and reached its lowest point within $6 \mathrm{~h}$ (Fig. 2a). Therefore, $6 \mathrm{~h}$ of reoxygenation injury following OGD was selected to simulate endothelial I/R injury in vitro. Fluorescence staining showed the mitochondrial net became short and fragmented after OGD/R injury, whereas ADV-HINT2 transfection at 10 and 30 MOI maintained mitochondrial length and morphology (Fig. 2b).
Further works confirmed that HINT2 transfection at 30 MOI modified dynamics-related molecules after OGD/R injury, as evidenced by reduced DRP1 and Fis 1 expression, inhibited DRP1 oligomerization and DRP1 phosphorylation at $\mathrm{Ser}^{616}$, increased MFN1 and MFN2 expression, and enhanced DRP1 phosphorylation at $\mathrm{Ser}^{637}$ (Fig. 2c, d and Fig. S2c). Similar results were observed in the 10 MOItransfected cells, except the reduction in MFN1 and MFN2 was not rescued (Fig. 2c, d and Fig. S2c), indicating that HINT2 overexpression was more prone to alleviate DRP1/ Fis1-regulated mitochondrial fission. Then, gain- and lossof-function assays of mitochondrial fission were conducted. Mdivi-1, a DRP1-specific inhibitor, increased mitochondrial length and cell viability, whereas FCCP, a fission enhancer, abolished the beneficial actions of HINT2 in sustaining the mitochondrial network and cell survival (Fig. S2d, e). Altogether, the above data demonstrated that HINT2 is necessary for balancing mitochondrial dynamics in CMECs suffering reperfusion/reoxygenation injury.

\section{HINT2-mediated inhibition of mitochondrial fission protected CMECs against reoxygenation-induced mitochondrial dysfunction}

Attempts were then made to identify whether the inhibitory effects of HINT2 on mitochondrial fission would protect mitochondrial function. Depolarized MMP is an early marker of mitochondrial dysfunction and apoptosis. MMP was significantly reduced after OGD/R injury, whereas HINT2 overexpression or Mdivi-1 treatment alleviated this reduction (Fig. 3a). However, regaining fission with FCCP antagonized the protective effects of HINT2 on MMP collapse (Fig. 3a). OGD/R injury resulted in a mitochondrial ROS (mtROS) burst and cellular ROS accumulation, whereas ADV-HINT2 transfection or Mdivi-1 treatment suppressed the mtROS burst and cellular ROS accumulation (Fig. 3b). Mn-SOD is one of the most forceful ROS scavengers in mitochondria. After OGD/R injury, the expression of Mn-SOD was significantly reduced, and this effect was alleviated by HINT2 overexpression or Mdivi-1 treatment (Fig. 3c). In addition, HINT2 overexpression or Mdivi-1 treatment suppressed the carbonyl modification of mitochondrial proteins caused by ROS toxicity (Fig. 3d). It can be predicted that regaining fission abrogated the suppressive effects of HINT2 on ROS accumulation, Mn-SOD down-regulation, and mitochondrial protein carbonylation (Fig. 3b-d). Taken together, these data confirmed that the enhanced mitochondrial function induced by HINT2 was highly associated with its role in inhibiting mitochondrial fission. 

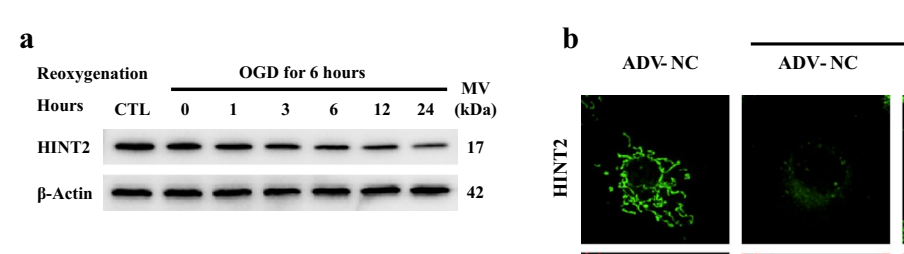

OGD/R injury
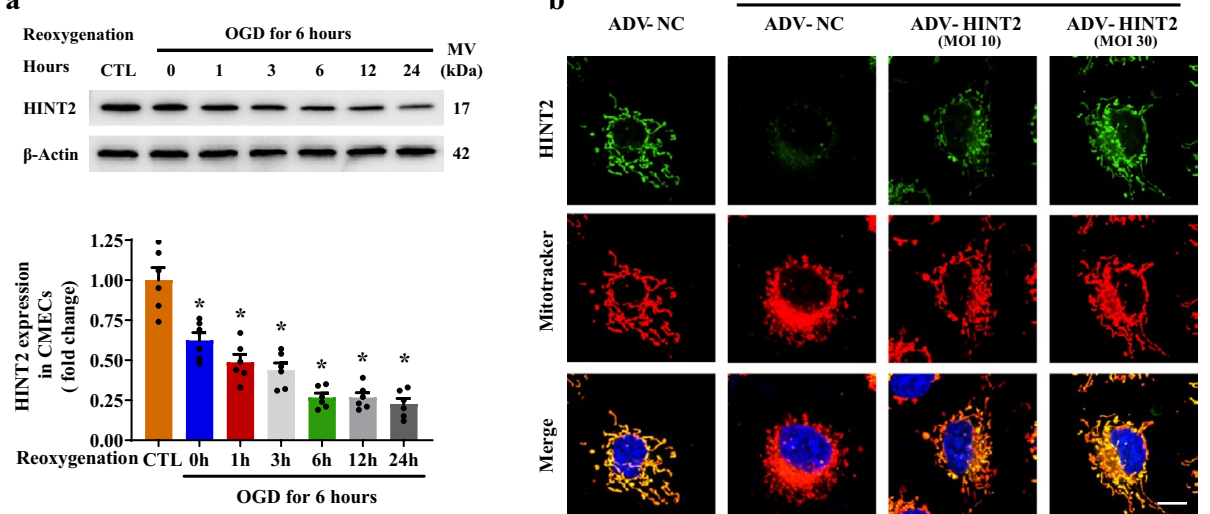

aDV-NC

- ADV-HINT2(MOI 10)

aDV-HINT2(MOI 30)
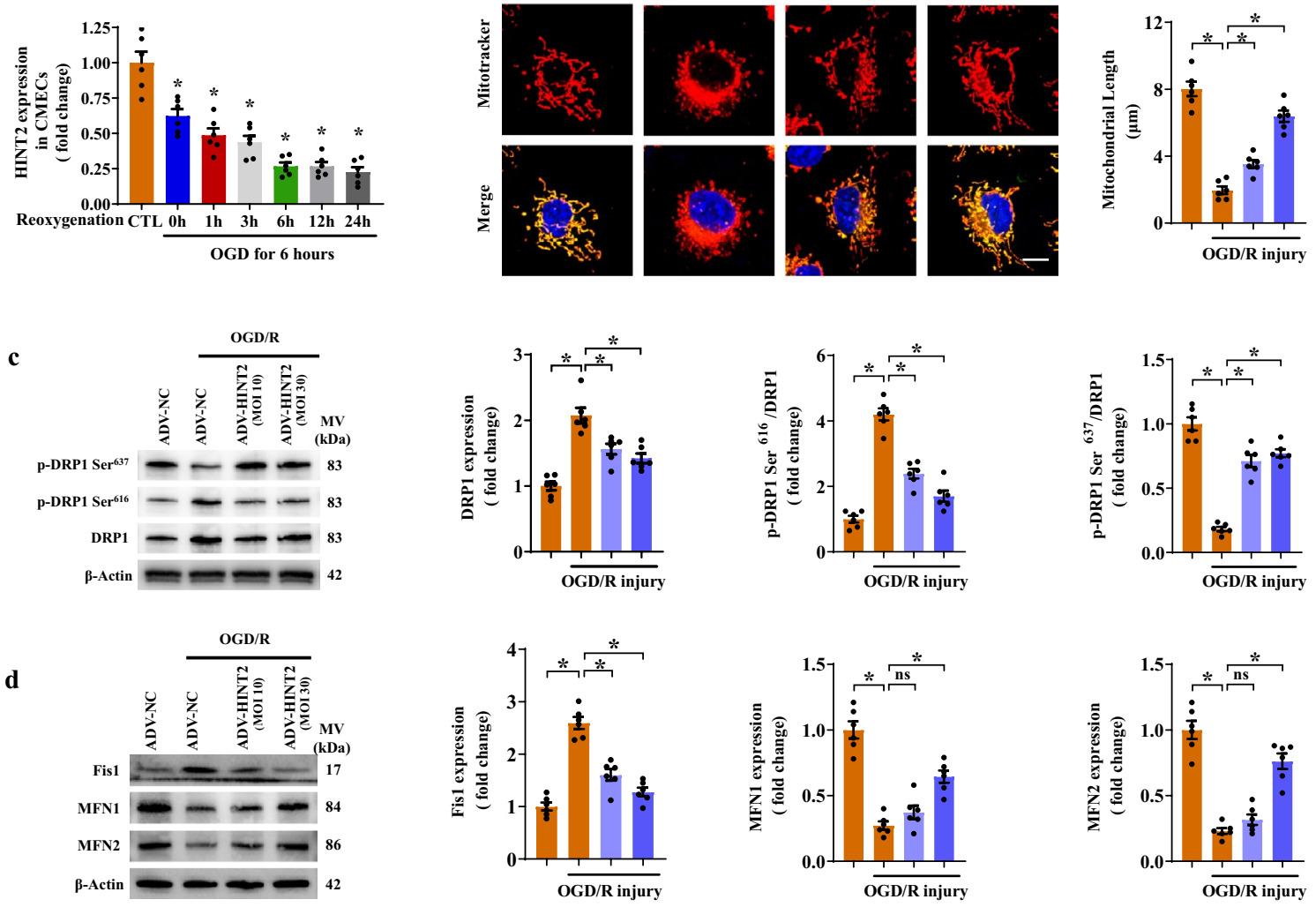

Fig. 2 HINT2 overexpression balanced mitochondrial dynamics in CMECs suffering from OGD/R injury. CMECs were isolated from 6- to 8-week-old WT mice and subjected to 6 h of OGD injury and 0-24 h of reoxygenation injury; the expression of HINT2 was measured by Western blot analysis (a). b-d Isolated CMECs were transfected with ADV-NC or ADV-HINT2, and then subjected to $6 \mathrm{~h}$ of OGD injury and $6 \mathrm{~h}$ of reoxygenation injury. HINT2 expression and mitochondrial morphology were evaluated by immunofluorescence

\section{Overexpression of HINT2 suppressed mitochondrion-dependent apoptosis after OGD/R injury}

Enhanced mitochondrial fission resulted in cytochrome $\mathrm{C}$ release, which further activated the mitochondriondependent apoptosis pathway. After OGD/R injury, cytochrome $\mathrm{C}$ was released from mitochondria to the cytoplasm in CMECs, as evidenced by the decreased colocalization of mitochondria and cytochrome $\mathrm{C}$ (Fig. 4a). Inhibition of fission with Mdivi-1 or HINT2 overexpression restricted cytochrome $\mathrm{C}$ release (Fig. 4a). Following cytochrome $\mathrm{C}$ release, the expression of $\mathrm{Bax}$ was upregulated and Bcl-2 was downregulated (Fig. 4b),

staining, and mitochondrial length was analyzed. Scale bars: $10 \mu \mathrm{m}$ (b). DRP1 expression, DRP1 phosphorylation at Ser ${ }^{616}$, and DRP1 phosphorylation at $\mathrm{Ser}^{637}$ were detected by Western blot analysis (c). Western blot analysis of the protein expression of Fis1, MFN1 and MFN2 (d). ${ }^{*} P<0.05$, significantly different as indicated. $n s$ Not significant. Biological replicates were performed for six times in the above studies

which was accompanied by enhanced expression cleavedcaspase 3 and caspase 9 (Fig. 4c), indicating that mitochondrion-dependent apoptosis was completely activated. In contrast, Mdivi-1 treatment or HINT2 overexpression suppressed the activation of the mitochondrion-dependent apoptosis pathway (Fig. 4b, c). Moreover, TUNELpositive CMECs were reduced by Mdivi-1 treatment or HINT2 overexpression, indicating that OGD/R injuryinduced apoptosis was suppressed (Fig. 4d and Fig. S2f). However, enhancing mitochondrial fission once more by FCCP abolished all the antiapoptotic effects of HINT2 elaborated above (Fig. 4a-d and Fig. S2f). 
a

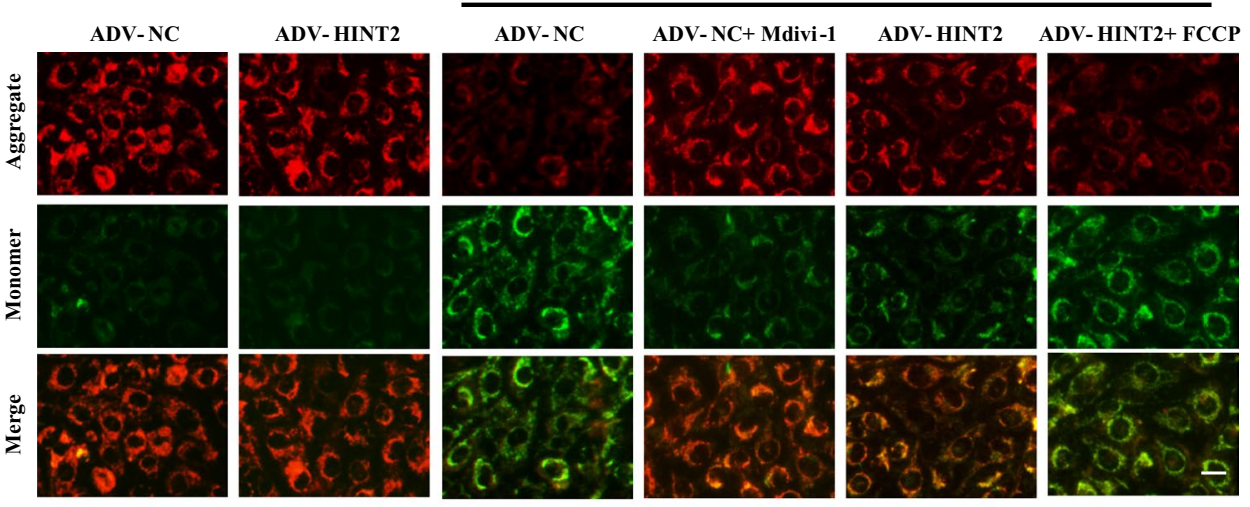

ADV-NC $\quad$ ADV-NC+Mdivi-1 - ADV-HINT2 (MOI 30) ADV-HINT2+FCCP

b
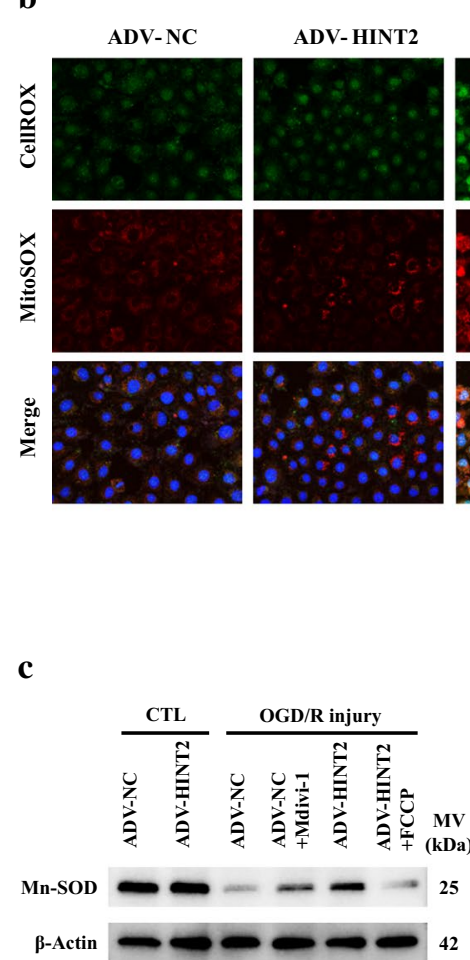
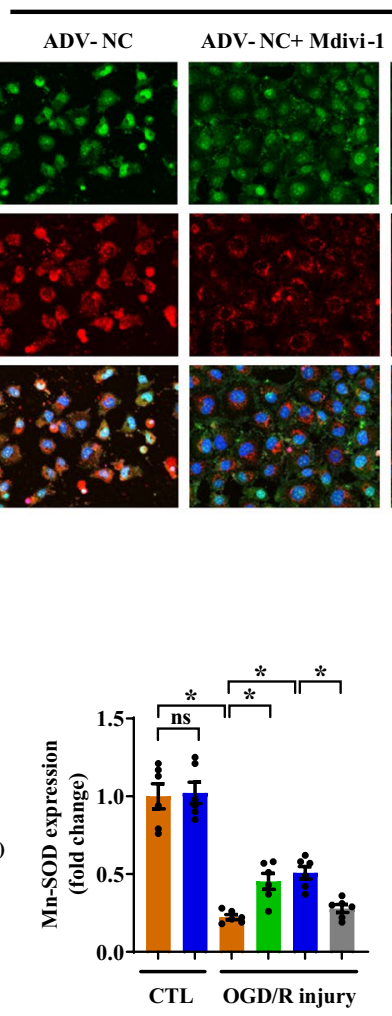

GD / R injury
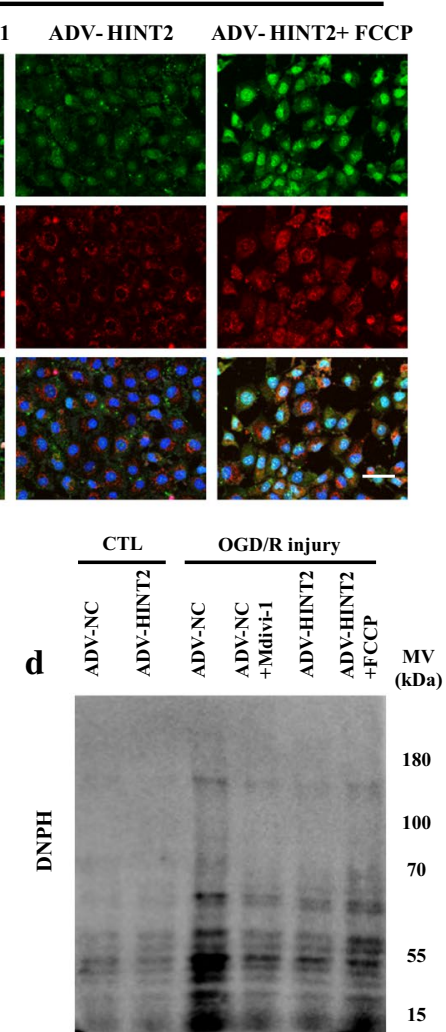
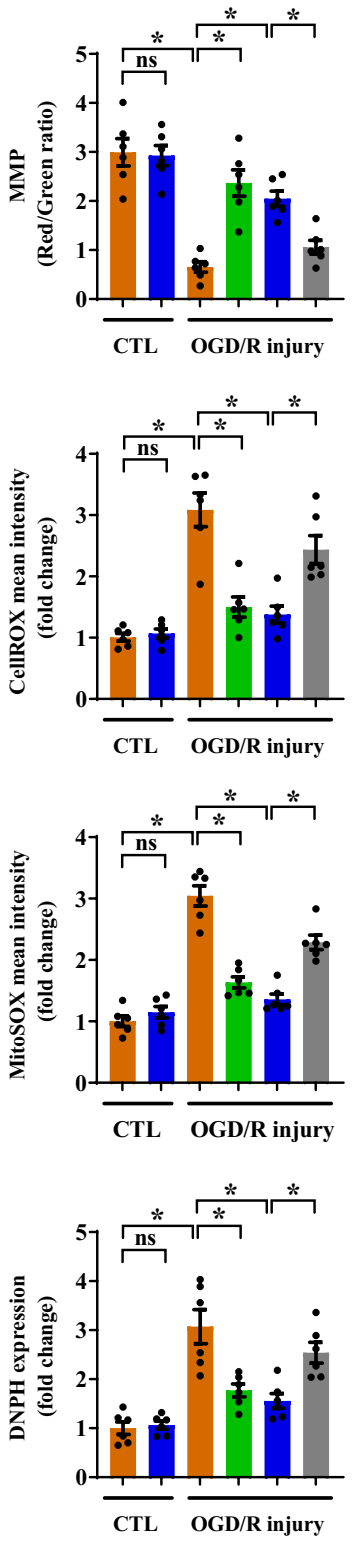

Fig. 3 HINT2 overexpression alleviated OGD/R injury-induced mitochondrial dysfunction by restraining mitochondrial fragmentation. CMECs were isolated from 6- to 8-week-old WT mice, transfected with ADV-NC or ADV-HINT2, and subjected to OGD/R injury. Mdivi-1 $(5 \mu \mathrm{M})$ was added to the ADV-NC group during OGD/R injury to inhibit mitochondrial fission. FCCP $(1 \mu \mathrm{M})$ was applied to ADV-HINT2 cells during OGD/R injury for $2 \mathrm{~h}$ to reintroduce mitochondrial fission. Mitochondrial membrane potential was measured using JC-1 fluorescence probe. Scale bars: $20 \mu \mathrm{m}$ (a). Cellular

\section{HINT2 inhibited mitochondrial calcium inflow by directly binding $M C U$ in $C M E C s$ under $O G D / R$ injury}

ROS and mitochondrial ROS were stained with CellROX (green) and MitoSOX (red), respectively. Scale bars: $60 \mu \mathrm{m}$ (b). Mn-SOD expression was detected by Western blot analysis (c). Protein lysates were incubated with DNPH to label carbonyl groups, and protein carbonylation was detected by Western blot analysis using anti-DNP antibodies (d). $* P<0.05$, significantly different as indicated. $n s$ Not significant. Biological replicates were performed for six times in the above studies

Calcium overload is involved in I/R injury-induced mitochondrial fission/apoptosis, and HINT2 has been suggested to modulate $\left[\mathrm{Ca}^{2+}\right]_{\mathrm{m}}$ inflow. Therefore, we hypothesized that the suppressive effect of HINT2 on mitochondrial 
$\mathbf{a}$

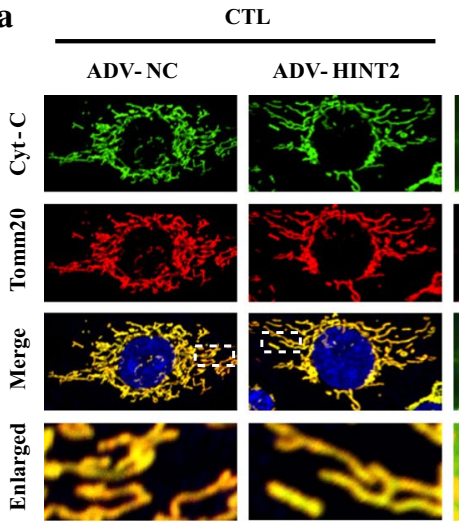

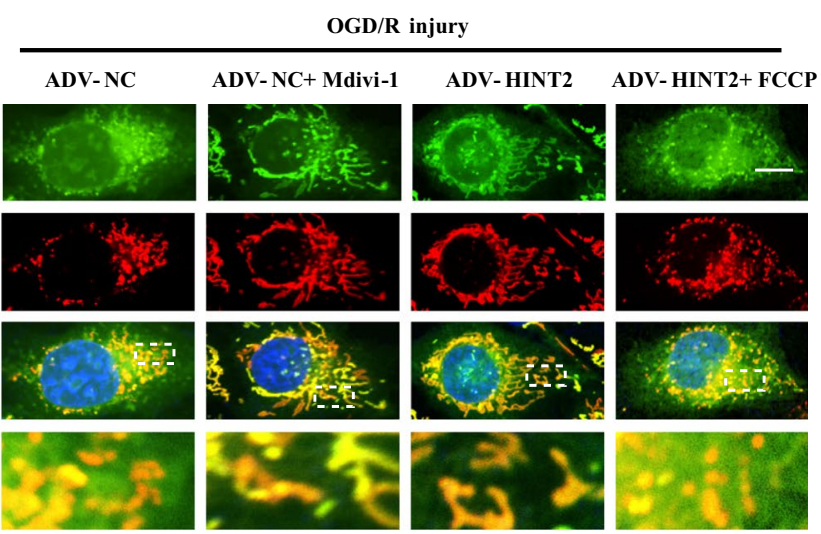
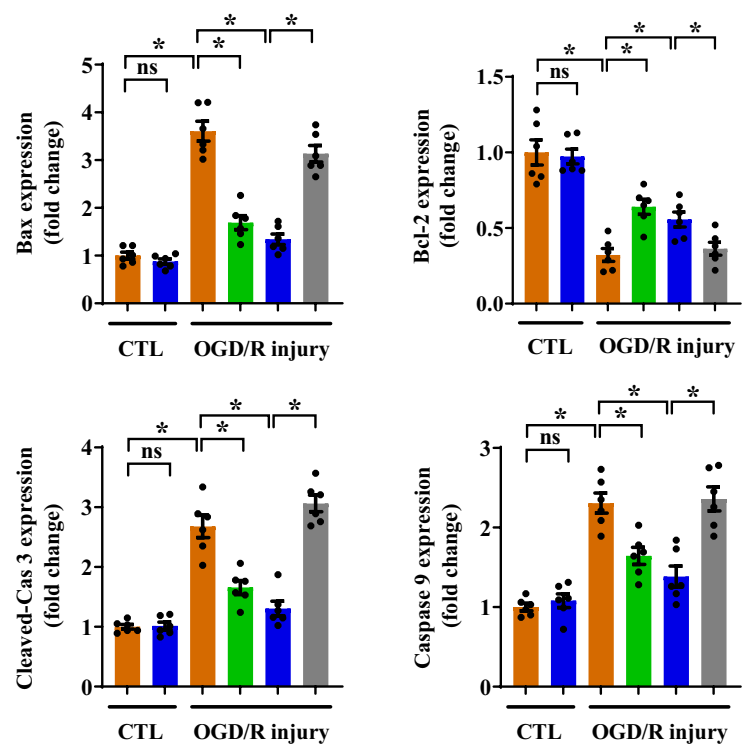

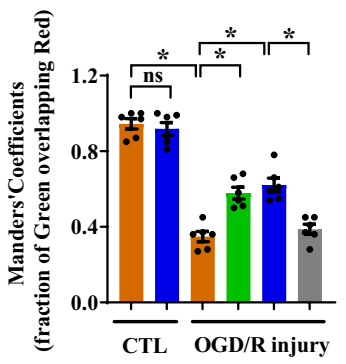

b
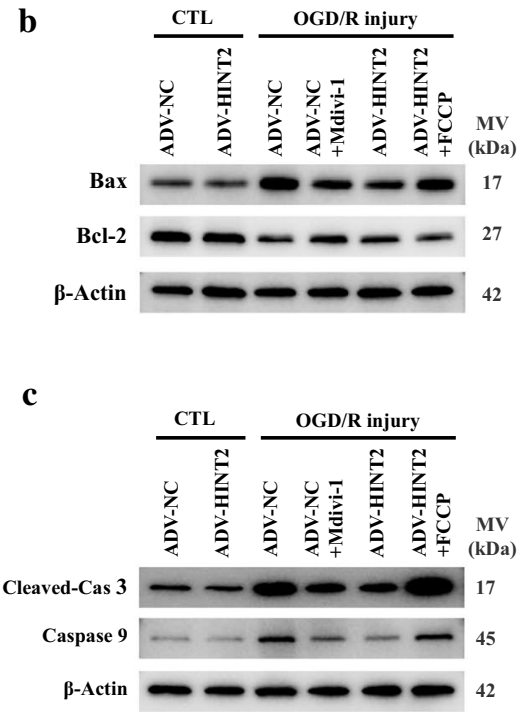

Fig. 4 HINT2 overexpression inhibited the mitochondrion-dependent apoptosis signaling pathway activated by OGD/R injury. CMECs were isolated from 6- to 8-week-old WT mice, transfected with ADV-NC or ADV-HINT2, and subjected to OGD/R injury. Mdivi-1 $(5 \mu \mathrm{M})$ was added to the ADV-NC group during OGD/R injury to inhibit mitochondrial fission. FCCP $(1 \mu \mathrm{M})$ was applied to ADVHINT2 cells during OGD/R injury for $2 \mathrm{~h}$ to reintroduce mitochondrial fission. The analysis of colocalization between mitochondria and cytochrome $\mathrm{C}$ was performed by immunostaining of cytochrome $\mathrm{C}$ (green) and Tomm20 (red). Cyt-C: cytochrome C. Scale bars: $10 \mu \mathrm{m}$ (a). Western blot analysis of the protein expression of Bax and Bcl-2 (b). Western blot analysis of the protein expression of cleavedCaspase 3 and Caspase 9. Cleaved-Cas 3: cleaved-Caspase 3 (c). Cell apoptosis was detected by TUNEL staining and quantified (d). $* P<0.05$, significantly different as indicated. $n s$ Not significant. Biological replicates were performed for six times in the above studies fission/apoptosis in endothelial cells was associated with $\left[\mathrm{Ca}^{2+}\right]_{\mathrm{m}}$ overload. First, our data indicated that lowering $\left[\mathrm{Ca}^{2+}\right]_{\mathrm{m}}$ exerted inhibitory effects on mitochondrial fission. Restricting $\left[\mathrm{Ca}^{2+}\right]_{\mathrm{m}}$ influx by Ru360, a specific MCU inhibitor, inhibited mitochondrial fission under OGD/R injury by suppressing DRP1 phosphorylation at $\mathrm{Ser}^{616}$ and DRP1 oligomerization (Fig. S3a-c). Then, further work demonstrated HINT2 overexpression significantly downregulated $\left[\mathrm{Ca}^{2+}\right]_{\mathrm{i}}$ and $\left[\mathrm{Ca}^{2+}\right]_{\mathrm{m}}$ levels under reoxygenation conditions (Fig. 5a). $\left[\mathrm{Ca}^{2+}\right]_{\mathrm{m}}$ homeostasis is largely dependent on the MCU complex. MCU was increased after reoxygenation injury but was reduced by HINT2 overexpression (Fig. 5b). In contrast, MICU1 and MICU2 were decreased after reoxygenation and maintained by HINT2 overexpression (Fig. 5b). Moreover, MCUb was significantly increased after reoxygenation, and HINT2 further enhanced MCUb expression (Fig. 5b). Co-IP assay showed that HINT2 could directly bind to MCU (Fig. 5c), and 4 AAs in HINT2 were identified as potential binding sites by structure-based protein interaction interface analysis (Fig. S3d). All AAs are within the domain of HINT2 but out of transit peptides that targeting mitochondria. Leu76, Phe78, and Asp80 are adjacent, while Trp160 is far from the other three AAs in spatial position. Based on the above results, Leu76, Phe78, and Asp80 were mutated into Ala in HINT2 mutant (Fig. S3e). MMGBSA analysis showed that HINT2 mutant had higher BFE with MCU 
a

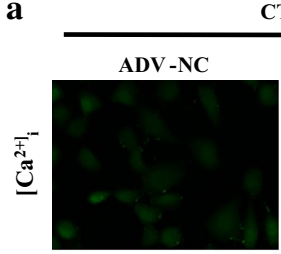

CTL
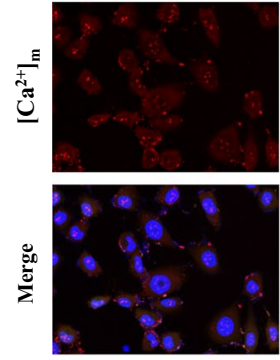
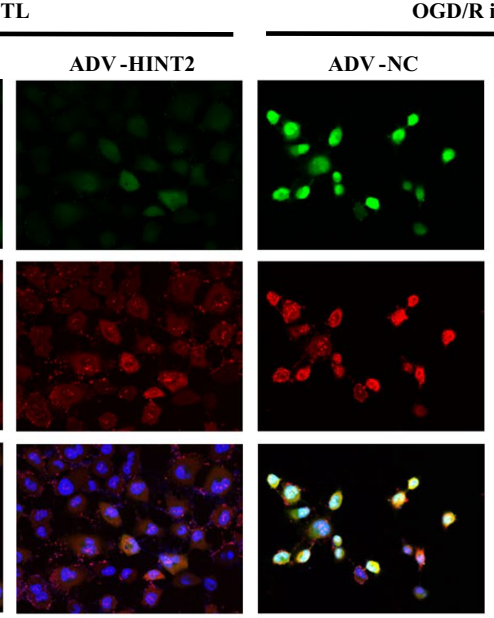
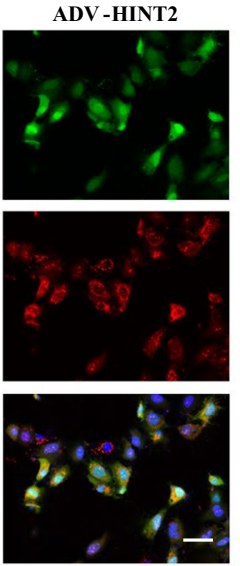

ADV-NC ADV-HINT2
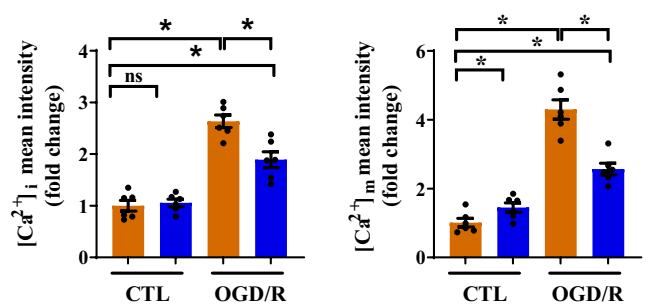
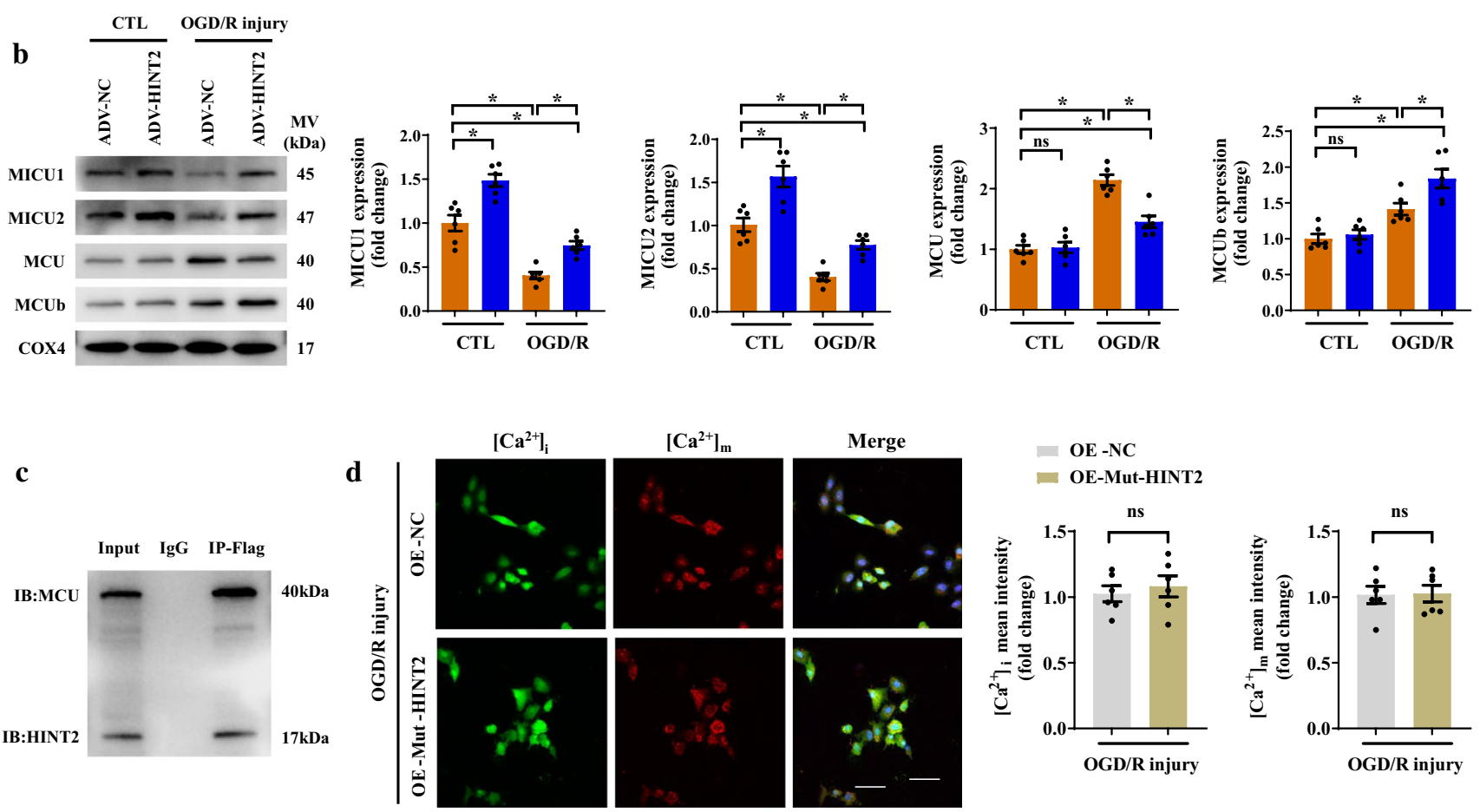

Fig. 5 HINT2 maintained mitochondrial calcium homeostasis by directly binding the MCU complex. CMECs were isolated from 6to 8-week-old WT mice, transfected with ADV-NC or ADV-HINT2, and subjected to OGD/R injury. $\left[\mathrm{Ca}^{2+}\right]_{\mathrm{i}}$ and $\left[\mathrm{Ca}^{2+}\right]_{\mathrm{m}}$ were stained with Fluo-4 AM (green) and Rhod-2 (red), respectively. Scale bars: $40 \mu \mathrm{m}$ (a). Western blot analysis of the protein expression of MICU1, MICU2, MCU, and MCUb (b). Co-IP analysis of MCU and Flag-

than wild type (Fig. S3f). Therefore, a plasmid containing HINT2 mutant was constructed and transfected into CMECs. Although HINT2 mutant still colocalized with MCU in mitochondria (Fig. S3g), the direct interaction between HINT2 and MCU was interrupted, as shown by the Co-IP assay (Fig. S3h), suggesting that the binding site in HINT2 was located within Leu76, Phe78, and Asp80. Additionally, overexpression of HINT2 mutant failed to inhibit calcium tagged HINT2 in CMECs (c). CMECs were transfected with plasmids containing HINT2 mutant or the negative control, and subjected to OGD/R injury. $\left[\mathrm{Ca}^{2+}\right]_{\mathrm{i}}$ and $\left[\mathrm{Ca}^{2+}\right]_{\mathrm{m}}$ were stained with Fluo-4 AM (green) and Rhod-2 (red), respectively. Mut-HINT2: HINT2 mutant. NC: negative control. Scale bars: $40 \mu \mathrm{m}(\mathbf{d}) . * P<0.05$, significantly different as indicated. $n s$ Not significant. Biological replicates were performed for 3-6 times in the above studies

overload and mitochondrial fragmentation in CMECs under OGD/R injury (Fig. 5d and Fig. S3i). Taken together, these data established that HINT2 stabilizes $\left[\mathrm{Ca}^{+}\right]_{\mathrm{m}}$ homeostasis by directly binding MCU and modulating the MCU complex, thereby inhibiting excessive mitochondrial fission in CMECs suffering OGD/R injury. 


\section{Overexpressing HINT2-alleviated CMEC functions after OGD/R injury by mitigating mitochondrial calcium overload}

Attempts were then made to determine whether HINT2 improves CMEC functions via $\mathrm{MCU}$ and $\left[\mathrm{Ca}^{2+}\right]_{\mathrm{m}}$ inhibition. After OGD/R injury, CMEC migration and tube formation ability were obviously impaired, the effects of which were largely rectified by HINT2 overexpression or Ru360 (Fig. S4a-c). Endothelial migration and angiogenesis are mediated mainly by the VEGF pathway. Ru360 treatment or HINT2 overexpression enhanced VEGFR2 expression and further upregulated VEGF signaling in OGD/R-injured CMECs (Fig. S4d). However, these contributions of HINT2 to facilitate cell migration, angiogenesis, and VEGF pathway activation were largely abolished after enhancing
$\left[\mathrm{Ca}^{2+}\right]_{\mathrm{m}}$ entry by spermine, an MCU activator (Fig. S4a-d). In addition, Ru360 treatment or HINT2 overexpression restrained the leakage of FITC-BSA from the impaired endothelial monolayer after OGD/R injury by enhancing VE-Cadherin junctions and VE-Cadherin/ $\beta$-Catenin interaction (Fig. 6a, b and Fig. S4e). However, these beneficial effects of HINT2 on maintaining endothelial barrier function were completely negated after the reintroduction of excessive $\left[\mathrm{Ca}^{2+}\right]_{\mathrm{m}}$ (Fig. 6a, b and Fig. S4e). After OGD/R injury, eNOS expression, eNOS phosphorylation and nitrite content were decreased, indicating the potential for less NO to be released from CMECs (Fig. 6c, d). Ru360 treatment or HINT2 overexpression significantly increased nitrite content in culture medium, along with increased eNOS expression and eNOS phosphorylation (Fig. 6c, d). However, the beneficial effects of HINT2 on nitrite levels, eNOS expression, $\mathbf{a}$
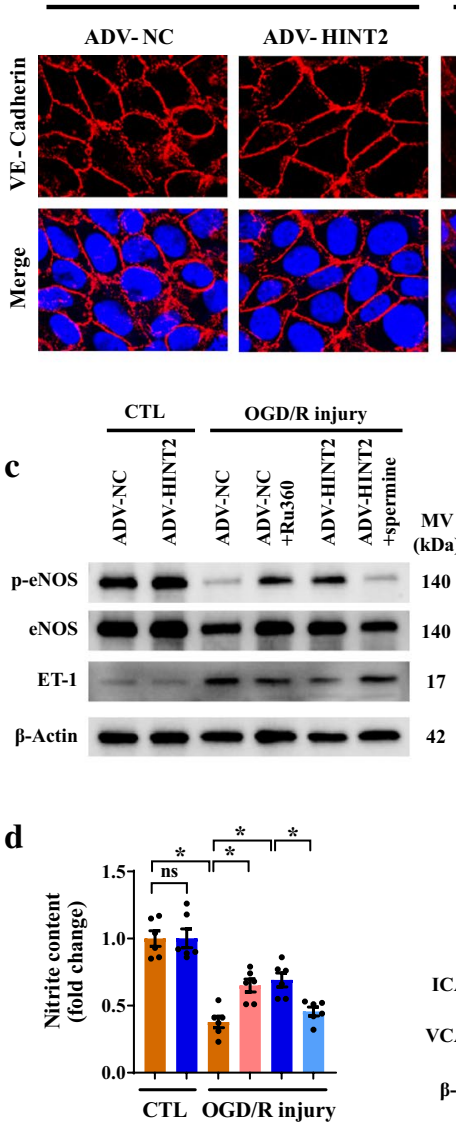

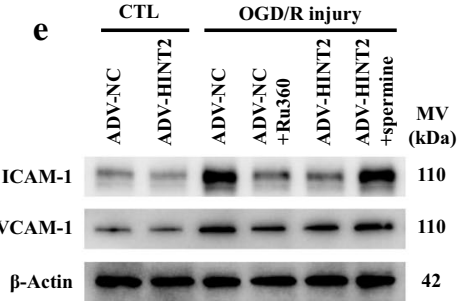

OGD/R injury
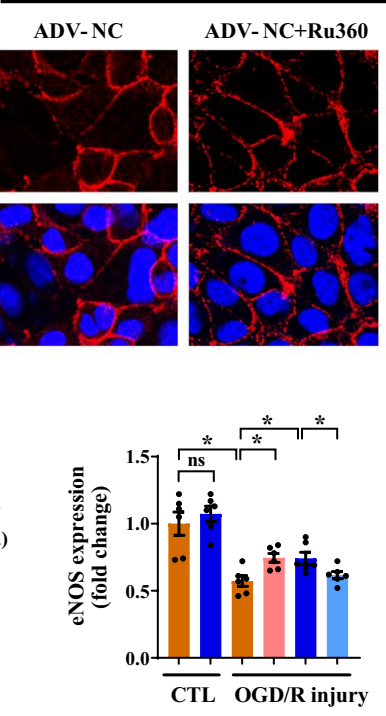

42
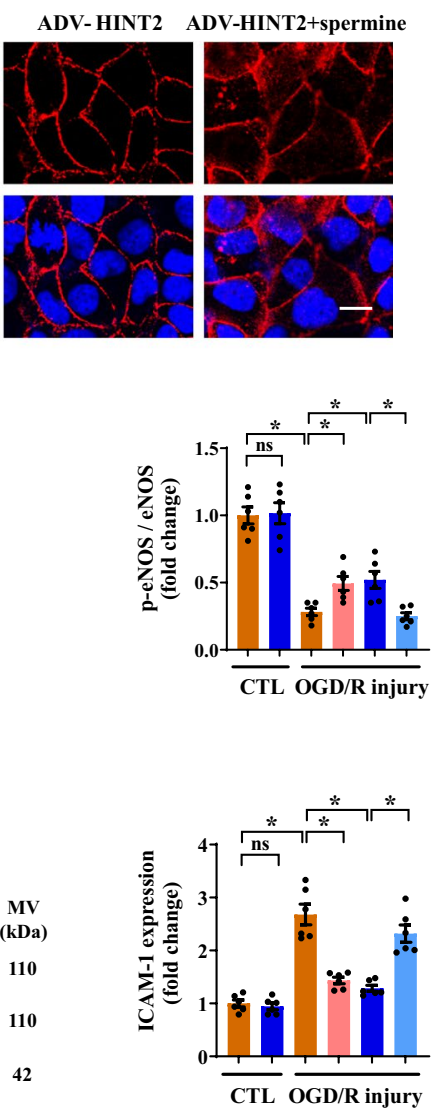

ADV-NC ADV-NC+Ru360 - ADV-HINT2 ADV-HINT2+spermine
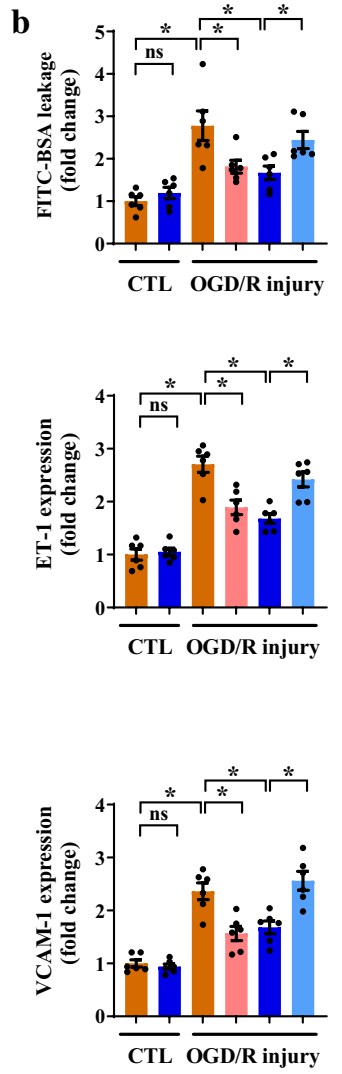

Fig. 6 Enhancing mitochondrial calcium influx negated the protective effects of HINT2 on CMECs in vitro. CMECs were isolated from 6- to 8-week-old WT mice, transfected with ADV-NC or ADVHINT2, and subjected to OGD/R injury. Cells transfected with ADV$\mathrm{NC}$ were pretreated with Ru360 $(10 \mu \mathrm{M}) 1 \mathrm{~h}$ before OGD/R injury to inhibit the MCU complex. Spermine $(10 \mu \mathrm{M})$ was added to ADVHINT2-infected cells during OGD/R injury for $2 \mathrm{~h}$ to reactivate the MCU complex. Endothelial junction was shown by immunofluores- cence staining of VE-Cadherin (red). Scale bars: $20 \mu \mathrm{m}$ (a). Endothelial monolayer permeability was evaluated by FITC-BSA leakage assay (b). eNOS expression, eNOS phosphorylation at $\mathrm{Ser}^{1177}$, and ET-1 expression were detected by Western blot analysis (c). Nitrite content in culture medium (d). Western blot analysis of the protein expression of ICAM-1 and VCAM-1 (e). $* P<0.05$, significantly different as indicated. $n s$ Not significant. Biological replicates were performed for six times in the above studies 
and eNOS phosphorylation were completely suppressed after spermine treatment (Fig. 6c, d). Moreover, spermine offset the inhibitory effects of HINT2 on ET-1 upregulation (Fig. 6c). Hypoxia plays a permissive role in motivating inflammatory response. Ru360 treatment or HINT2 overexpression decreased ICAM-1 and VCAM-1 expression in OGD/R-injured cells (Fig. 6e). It could be expected that the above anti-inflammatory actions of HINT2 were completely abolished after regaining $\left[\mathrm{Ca}^{2+}\right]_{\mathrm{m}}$ overload (Fig. 6e). In summary, the above results confirmed that HINT2 preserved angiogenic potential, strengthened endothelial barrier function, stimulated eNOS activation, and inhibited inflammatory response in CMECs exposed to reperfusion injury in vitro, at least in part due to its role in maintaining calcium homeostasis.

\section{Reactivation of the MCU complex abolished the protective effects of HINT2 overexpression on cardiac microvascular functions in I/R mice}

To gain more convincing evidence that the MCU complex was a downstream target of HINT2 in cardiac microvascular protection, the effects of Ru360 and spermine were investigated in mice. Similar to HINT2 overexpression, Ru360 treatment alleviated the no-reflow phenomenon and maintained microvascular perfusion in ischemia hearts, as evidenced by increased thioflavin S perfusion, more FITCperfused microvessels, enhanced eNOS phosphorylation, suppressed ET-1 expression, and higher nitrite content (Fig. 7a-d). However, in the presence of spermine treatment, HINT2 overexpression failed to alleviate the no-reflow phenomenon and enhance microvascular perfusion (Fig. 7a-d). As we have shown previously, HINT2 overexpression reduced VE-Cadherin phosphorylation and strengthened the VE-Cadherin/ $\beta$-Catenin interaction, thereby inhibiting Evans blue leakage from cardiac microcirculation (Fig. S5a-c). However, these improvements were not observed in the AAV-HINT2 group pretreated with spermine (Fig. S65c). Moreover, spermine-mediated MCU complex activation abrogated the suppressive effects of HINT2 on VCAM-1 and ICAM-1 expression (Fig. S5d). Importantly, blocking the MCU complex with Ru360 limited the myocardial infarction size, whereas activating the MCU complex nullified the protective effects of HINT2 on infarction expansion (Fig. 7e). In conclusion, the above in vivo data further explicitly showed that the protective effects of HINT2 relied on the inhibition of the MCU complex and $\left[\mathrm{Ca}^{2+}\right]_{\mathrm{m}}$ overload.

\section{Discussion}

The cardiac microcirculation is particularly vulnerable to the detrimental effects of reperfusion and has been recognized as a therapeutic target for cardiac I/R injury. The present data demonstrated that HINT2 overexpression protected the cardiac microcirculation against I/R injury by enhancing blood perfusion, barrier function, and anti-inflammatory action. The protection from HINT2 was attributed to its role in regulating MCU complex function and maintaining $\left[\mathrm{Ca}^{2+}\right]_{\mathrm{m}}$ homeostasis in CMECs. HINT2 overexpression inhibited $\left[\mathrm{Ca}^{2+}\right]_{\mathrm{m}}$ overload-evoked mitochondrial fission and, thereby, maintained mitochondrial function, and inhibited the mitochondrion-dependent apoptotic pathway, ultimately improving endothelial survival and function in response to reoxygenation injury (Scheme 1).

It is widely acknowledged that a timely reperfusion remedy is necessary for rescuing ischemic myocardium [22]. In contrast, after reperfusion, superabundant reactive species and inflammatory cascades burst rapidly and assault the cardiac microcirculation [29]. The most immediate consequence is cardiac edema and, if severe, slow-reflow and no-reflow phenomena, which discounted the benefits of coronary reperfusion strategies in MI patients [17]. The present study identified that HINT2 overexpression enhanced CMEC migration and angiogenesis by mediating the VEGF signaling pathway, which sustained CMEC survival in the setting of reoxygenation. In addition, HINT2 overexpression increased nitrite content, eNOS expression, and phosphorylation in the myocardium and CMECs, along with decreased ET-1 expression, indicating a protective role for HINT2 in the cardiac vasodilator response. Through the above mechanisms, HINT2 maintains microvascular density and increases blood reperfusion in the ischemic region. During the reperfusion stage, increased microvascular permeability and inflammatory exudation are the major contributors to cardiac edema. HINT2 overexpression strengthened VE-Cadherin/ $\beta$-Catenin interaction and suppressed VCAM-1 and ICAM-1 to enhance endothelial barrier function and prevent inflammatory response in both I/R-injured myocardium and OGD/R-injured CMECs. In combination with our previous study, these newly obtained data further support the beneficial role of HINT2 in cardioprotection, especially in microvascular structure and function during the reperfusion stage.

Long-term and substantially elevated $\left[\mathrm{Ca}^{2+}\right]_{\mathrm{m}}$ triggered MMP collapse, ROS overproduction, mPTP opening, and, most importantly, apoptosis in cardiomyocytes, which was identified as a core mechanism underlying cardiac I/R injury [40]. However, the pathological role of $\left[\mathrm{Ca}^{2+}\right]_{\mathrm{m}}$ in mitochondrial fission and endothelial functions in I/R injury was not explored in detail. The present study revealed that 
a

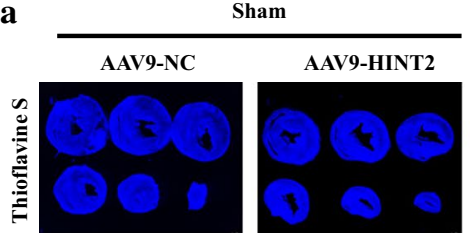

b

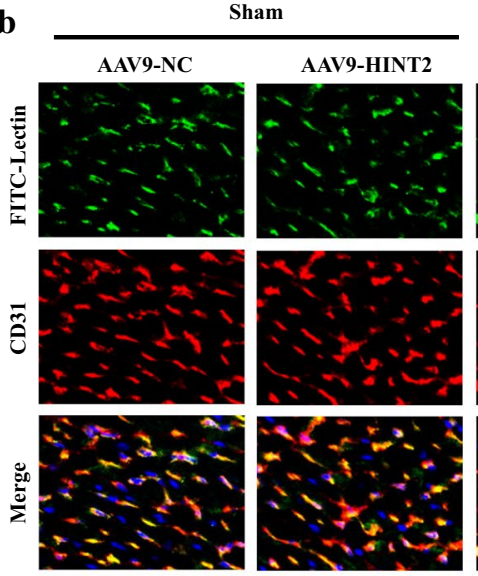

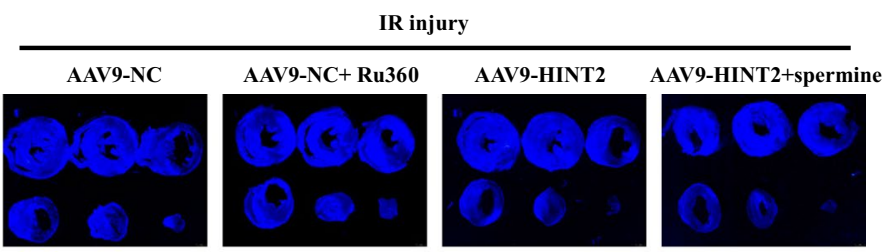

IR injury
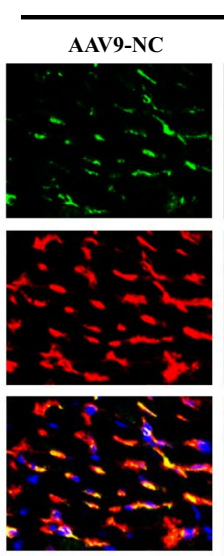

AAV9-NC+ Ru360
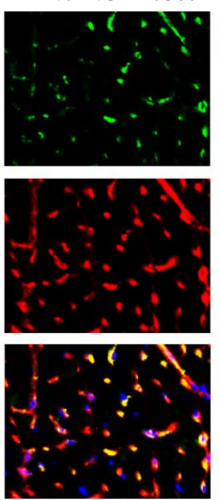

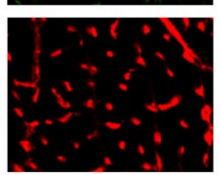

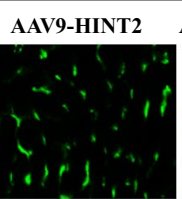

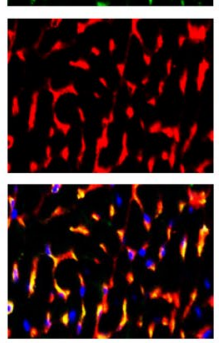

AAV9-NC

aAV9-HINT2

AAV9-NC+Ru360

- AAV9-HINT2+spermine
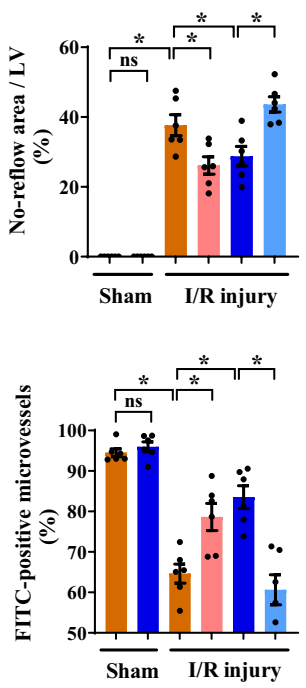

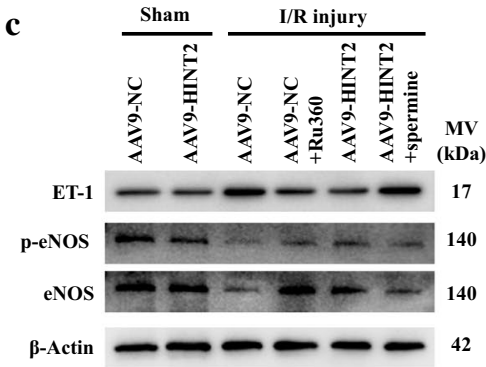

d

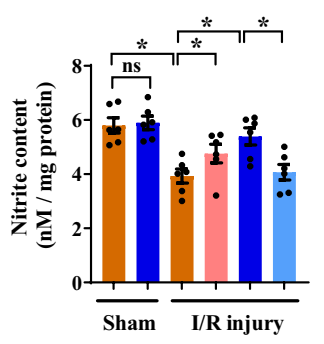

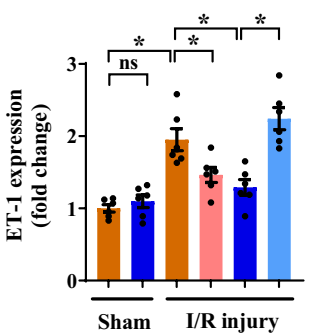

Sham+AAV9-NC

e

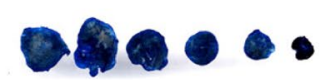

Sham+AAV9-HINT2

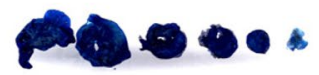

I/R+AAV9-NC

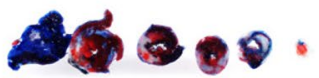

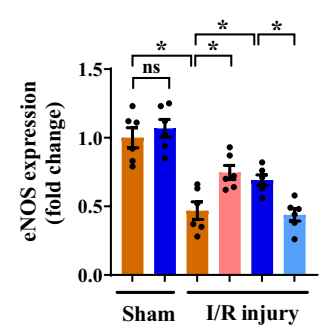

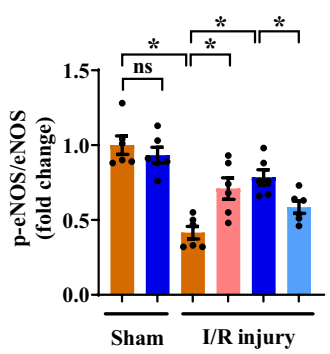

I/R+AAV9-NC+Ru360

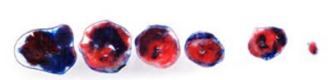

I/R+AAV9-HINT2

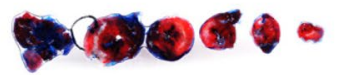

I/R+AAV9-HINT2+Spermine

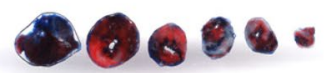

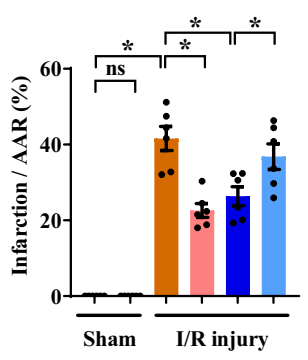

Fig. 7 Endothelial-specific overexpression of HINT2 protected the cardiac microcirculation against $\mathrm{I} / \mathrm{R}$ injury by modulating the $\mathrm{MCU}$ complex. Mice were transfected with AAV9-NC or AAV9-HINT2 and suffered from $45 \mathrm{~min}$ ischemia and $6 \mathrm{~h}$ reperfusion injury. Ru360 $(50 \mathrm{nmol} / \mathrm{kg}$ ) was injected intraperitoneally into AAV9-NC mice $1 \mathrm{~h}$ before I/R injury to inhibit the MCU complex. Spermine $(5 \mathrm{mg} / \mathrm{kg}$ ) was injected intraperitoneally into AAV9-HINT2 mice $1 \mathrm{~h}$ before I/R injury to activate the MCU complex. No reflow area after reperfusion was detected by thioflavin S staining and quantified (a). Cardiac

the restoration of $\left[\mathrm{Ca}^{2+}\right]_{\mathrm{m}}$ by Ru360 inhibited mitochondrial fission caused by OGD/R injury via inhibition of DRP1 phosphorylation and oligomers in CMECs. Moreover, microvascular perfusion was indicated by the ratio of lectin-positive microvessels (green) to CD31-positive microvessels (red). Scale bars: $20 \mu \mathrm{m}$ (b). ET-1 expression, eNOS expression, and eNOS phosphorylation at $\mathrm{Ser}^{1177}$ in left ventricle were detected by Western blot analysis (c). Nitrite content in left ventricle (d). Infarcted area was detected by TTC staining and quantified (e). ${ }^{*} P<0.05$, significantly different as indicated. $n s$ Not significant. Biological replicates were performed for six times in the above studies

fission inhibition favored the recovery of mitochondrial dysfunction and the suppression of the mitochondrial apoptosis pathway in OGD/R-injured CMECs. A few studies 
Scheme 1 Diagram of potential mechanism for HINT2 in repairing I/R-induced cardiac microvascular injury

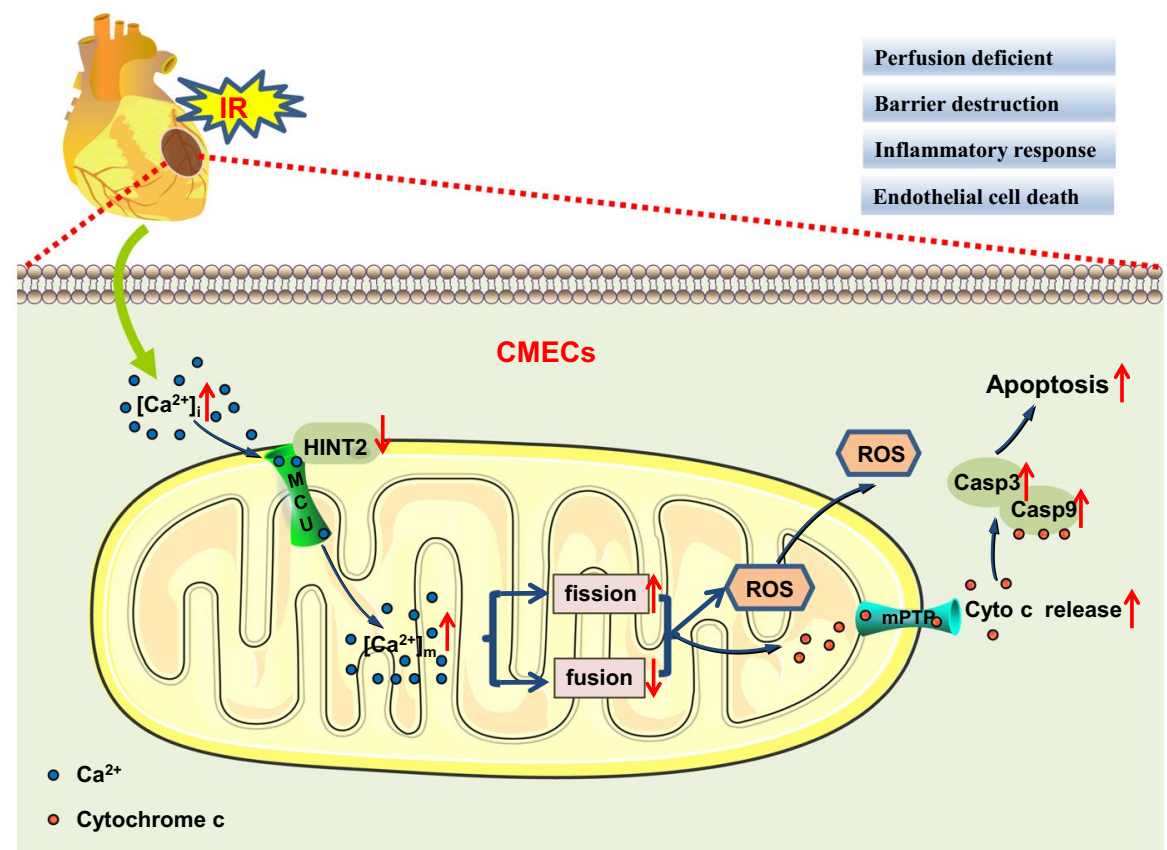

have been performed and proposed that the suppression of $\left[\mathrm{Ca}^{2+}\right]_{\mathrm{m}}$ overload maintains endothelial functions under I/R injury, hyperuricemia, and diabetes [20, 37, 45]. The present study demonstrated that the inhibition of $\left[\mathrm{Ca}^{2+}\right]_{\mathrm{m}}$ overload via HINT2 overexpression or Ru360 treatment protected CMECs from reoxygenation injury in terms of endothelial barrier collapse, eNOS reduction, inflammatory reaction, and angiogenic disorder. Additionally, our further attempts confirmed that the maintenance of $\left[\mathrm{Ca}^{2+}\right]_{\mathrm{m}}$ homeostasis relieved cardiac microvascular dysfunction induced by $\mathrm{I} / \mathrm{R}$ injury, as evidenced by decreased no-reflow area, increased reperfusion, enhanced barrier function, and reduced expression of adhesion molecules, all of which indispensably contributed to limiting infarction expansion.

$\left[\mathrm{Ca}^{2+}\right]_{\mathrm{m}}$ influx is principally mediated by the MCU complex, which is critical for mPTP opening in response to increased $\left[\mathrm{Ca}^{2+}\right]_{\mathrm{I}}[2]$. The identification of subunits in the MCU complex paves the way to determine the different roles of each subunit in cardiac I/R injury [24]. Four MCU subunits constitute the basic components of MCU complexes. Cardiomyocyte-specific deletion of MCU subunits or Ru360 treatment protected cardiomyocytes from $\left[\mathrm{Ca}^{2+}\right]_{\mathrm{m}}$ overload and mPTP opening and, therefore, decreased infarct size and preserved cardiac function in I/R hearts [15, 43]. Importantly, MCU inhibition in CMECs has been suggested to improve cardiac microcirculation after I/R injury [27]. MICU1 and MICU2 form heterodimers and act as MCU gatekeepers to keep the channel closed by sensing the $\left[\mathrm{Ca}^{2+}\right]_{\mathrm{i}}$ concentration [38]. Restraining $\left[\mathrm{Ca}^{2+}\right]_{\mathrm{m}}$ influx via the upregulation of MICU1 protected the heart from I/R injury [3, 50]. MCUb was not expressed at baseline, but was upregulated when sensing calcium elevation in the cytoplasm [21]. MCUb overexpression reduced mPTP opening and decreased infarct size following IR injury [21, 26]. Although the function of MCU in the cardiac microcirculation was investigated before, other subunits were ignored in this research field. HINT2 was introduced only to modulate the MCU complex in pancreatic cancer, sensitizing tumor cells to apoptosis by enhancing $\left[\mathrm{Ca}^{2+}\right]_{\mathrm{m}}$ uptake [6]. The current work showed that HINT2 overexpression inhibited the upregulation of MCU, reversed the reduction in MICU1 and MICU2, and further increased MCUb expression in CMECs under OGD/R injury. A protein-protein interaction study identified Leu76, Phe78, and Asp80 as sites for HINT2 to directly combine with MCU in CMECs. The above results indicated that HINT2 exerted protective benefits on endothelial cells by directly binding MCU and thereby regulating the MCU complex and $\left[\mathrm{Ca}^{2+}\right]_{\mathrm{m}}$ homeostasis in the setting of reoxygenation injury.

Several potential biases or imprecisions should be considered. Unspecific transfection of AAV9-HINT2 had only been excluded in cardiomyocytes but not in other cell types, such as fibroblasts and smooth muscle cells. Smooth muscle cells are major components of arterioles and are in charge of vasodilatation. Based on the presented data, a role of HINT2 in vascular physiology is sensible, but the contribution from other cell types cannot be excluded. The present study used the FITC-lectin perfusion method as a surrogate parameter to estimate microvascular blood flow [31, 42]. More direct methods to determine coronary flow and reactive hyperemia of the coronary arteries during reperfusion would provide more precise conclusions [16, 23]. The 
short half-life of $\mathrm{NO}$ makes it difficult to directly detect $\mathrm{NO}$ content in the myocardium [32]. The present study detected nitrite by a commercial kit to indirectly reflect NO levels. It should be pointed out that nitrite is both a NO metabolite and a reserve for NO synthesis [34]. Therefore, it is hard to explain whether the increased nitrite by HINT2 overexpression could be interpreted as enhanced NO synthesis. However, increased nitrite has been reported to improve endothelial dysfunction and attenuate infarct size in myocardial IR injury [14], still supporting a protective role of HINT2 in cardiac microcirculation. Under normoxia, without affecting MCU protein levels, the expression of MICU1 and MICU2, as well as $\left[\mathrm{Ca}^{2+}\right]_{\mathrm{m}}$ level, was increased after HINT2 overexpression. Whether MICU1 and MICU2 could be directly bound by HINT2 and thereby modulate $\left[\mathrm{Ca}^{2+}\right]_{\mathrm{m}}$ influx was not identified. Future studies are warranted to test this hypothesis.

In conclusion, the present study determined that HINT2 acted as a protective factor in the cardiac microvasculature. HINT2 protected cardiac microvascular structure and function against I/R injury and maintained mitochondrial dynamics and function by modulating $\left[\mathrm{Ca}^{2+}\right]_{\mathrm{m}}$ through the MCU complex in CMECs. These findings elucidate a new role of HINT2 in cardioprotection and suggest that the MCU complex $/\left[\mathrm{Ca}^{2+}\right]_{\mathrm{m}}$ overload/mitochondrial fission/apoptosis signaling pathway is potentially one of the pathological mechanisms underlying microvascular I/R injury.

Supplementary Information The online version contains supplementary material available at https://doi.org/10.1007/s00395-021-00905-4.

Author contributions Study design: SL, JC, and YC. Experiment conduct: SL, ML, YW, and TM. Material preparation: JG, YX, MF, AC, DL, ES, and FX. Data collection and analysis: SL, YW, and YC. Drafting manuscript: SL, JC, and YC. Revising manuscript content: SL, JC, and YC. Funding and general supervision: ZC, JQ, and JG.

Funding This study was supported by the National Program on Key Basic Research Project of China (Grant Nos. 2019YFC0840601 and 2014CBA02003), National Natural Science Foundation of China (Grant Nos. 82170338, 81970295, 81800347, 81870267, 81521001, $81670318,81570314,81900307$, and 82000380), Grant of Shanghai Shenkang on Key Clinical Research Project (Grant Nos. SHDC2020CR2015A and SHDC12019104), Grant of Shanghai Science and Technology Committee (Grant Nos. 19MC1910300, 18411950200, and 20JC1410800), Key Medical and Health Projects of Xiamen Province (No. 3502Z20204004), Grant of Shanghai Municipal Commission of Health and Family Planning (Grant No. 2017YQ057), Grant of Zhongshan Hospital Affiliated to Fudan University (Grant No. 2018ZSLC01), VG Funding of Clinical Trials (2017-CCA-VG-036), and Merck Funding (Xinxin-merck-fund-051).

Data availability The datasets used and analyzed supporting the findings of this study are available in this paper or the Supplementary Materials. Any other raw data generated or analyzed in this study can be obtained upon reasonable request from the corresponding authors.

\section{Declarations}

Conflict of interest The authors declare that they have no competing interests.

Ethical approval All animal study protocols were approved by the Ethics Committee for Animal Experiments and strictly adhered to the guidelines for animal experiments of Fudan University (Shanghai, China). The registration number of the ethical approval is 2019-186.

Open Access This article is licensed under a Creative Commons Attribution 4.0 International License, which permits use, sharing, adaptation, distribution and reproduction in any medium or format, as long as you give appropriate credit to the original author(s) and the source, provide a link to the Creative Commons licence, and indicate if changes were made. The images or other third party material in this article are included in the article's Creative Commons licence, unless indicated otherwise in a credit line to the material. If material is not included in the article's Creative Commons licence and your intended use is not permitted by statutory regulation or exceeds the permitted use, you will need to obtain permission directly from the copyright holder. To view a copy of this licence, visit http://creativecommons.org/licenses/by/4.0/.

\section{References}

1. Alevriadou BR, Shanmughapriya S, Patel A, Stathopulos PB, Madesh M (2017) Mitochondrial $\mathrm{Ca}(2+)$ transport in the endothelium: regulation by ions, redox signalling and mechanical forces. J R Soc Interface. https://doi.org/10.1098/rsif.2017.0672

2. Alevriadou BR, Patel A, Noble M, Ghosh S, Gohil VM, Stathopulos PB, Madesh M (2020) Molecular nature and physiological role of the mitochondrial calcium uniporter channel. Am J Physiol Cell Physiol. https://doi.org/10.1152/ajpcell.00502.2020

3. Banavath HN, Roman B, Mackowski N, Biswas D, Afzal J, Nomura Y, Solhjoo S, O'Rourke B, Kohr M, Murphy E, Steenbergen C, Das S (2019) miR-181c activates mitochondrial calcium uptake by regulating MICU1 in the heart. J Am Heart Assoc 8:e012919. https://doi.org/10.1161/jaha.119.012919

4. Bøtker HE, Hausenloy D, Andreadou I, Antonucci S, Boengler K, Davidson SM, Deshwal S, Devaux Y, Di Lisa F, Di Sante M, Efentakis P, Femminò S, García-Dorado D, Giricz Z, Ibanez B, Iliodromitis E, Kaludercic N, Kleinbongard P, Neuhäuser M, Ovize M, Pagliaro P, Rahbek-Schmidt M, Ruiz-Meana M, Schlüter KD, Schulz R, Skyschally A, Wilder C, Yellon DM, Ferdinandy P, Heusch G (2018) Practical guidelines for rigor and reproducibility in preclinical and clinical studies on cardioprotection. Basic Res Cardiol 113:39. https://doi.org/10.1007/s00395-018-0696-8

5. Brenner C, Bieganowski P, Pace HC, Huebner K (1999) The histidine triad superfamily of nucleotide-binding proteins. J Cell Physiol 181:179-187. https://doi.org/10.1002/(sici)10974652(199911)181:2\%3c179::Aid-jcp1\%3e3.0.Co;2-8

6. Chen L, Sun Q, Zhou D, Song W, Yang Q, Ju B, Zhang L, Xie H, Zhou L, Hu Z, Yao H, Zheng S, Wang W (2017) HINT2 triggers mitochondrial $\mathrm{Ca}(2+)$ influx by regulating the mitochondrial $\mathrm{Ca}(2+)$ uniporter (MCU) complex and enhances gemcitabine apoptotic effect in pancreatic cancer. Cancer Lett 411:106-116. https://doi.org/10.1016/j.canlet.2017.09.020

7. Chen Y, Li S, Zhang Y, Wang M, Li X, Liu S, Xu D, Bao Y, Jia P, Wu N, Lu Y, Jia D (2021) The lncRNA Malat1 regulates microvascular function after myocardial infarction in mice via 
miR-26b-5p/Mfn1 axis-mediated mitochondrial dynamics. Redox Biol 41:101910. https://doi.org/10.1016/j.redox.2021.101910

8. de Waha S, Patel MR, Granger CB, Ohman EM, Maehara A, Eitel I, Ben-Yehuda O, Jenkins P, Thiele H, Stone GW (2017) Relationship between microvascular obstruction and adverse events following primary percutaneous coronary intervention for STsegment elevation myocardial infarction: an individual patient data pooled analysis from seven randomized trials. Eur Heart J 38:3502-3510. https://doi.org/10.1093/eurheartj/ehx414

9. Dong Y, Undyala VVR, Przyklenk K (2016) Inhibition of mitochondrial fission as a molecular target for cardioprotection: critical importance of the timing of treatment. Basic Res Cardiol 111:59. https://doi.org/10.1007/s00395-016-0578-x

10. Elrod JW, Wong R, Mishra S, Vagnozzi RJ, Sakthievel B, Goonasekera SA, Karch J, Gabel S, Farber J, Force T, Brown JH, Murphy E, Molkentin JD (2010) Cyclophilin D controls mitochondrial pore-dependent $\mathrm{Ca}(2+)$ exchange, metabolic flexibility, and propensity for heart failure in mice. J Clin Invest 120:3680 3687. https://doi.org/10.1172/jci43171

11. Fan M, Chen Z, Huang Y, Xia Y, Chen A, Lu D, Wu Y, Zhang N, Zhang P, Li S, Chen J, Zhang Y, Sun A, Zou Y, Hu K, Qian J, Ge J (2020) Overexpression of the histidine triad nucleotide-binding protein 2 protects cardiac function in the adult mice after acute myocardial infarction. Acta Physiol (Oxf) 228:e13439. https://doi. org/10.1111/apha.13439

12. Gao E, Lei YH, Shang X, Huang ZM, Zuo L, Boucher M, Fan Q, Chuprun JK, Ma XL, Koch WJ (2010) A novel and efficient model of coronary artery ligation and myocardial infarction in the mouse. Circ Res 107:1445-1453. https://doi.org/10.1161/circr esaha. 110.223925

13. Granger DN, Kvietys PR (2017) Reperfusion therapy-What's with the obstructed, leaky and broken capillaries? Pathophysiology 24:213-228. https://doi.org/10.1016/j.pathophys.2017.09.003

14. Griffiths K, Lee JJ, Frenneaux MP, Feelisch M, Madhani M (2021) Nitrite and myocardial ischaemia reperfusion injury. Where are we now? Pharmacol Ther 223:107819. https://doi.org/10.1016/j. pharmthera.2021.107819

15. Guan L, Che Z, Meng X, Yu Y, Li M, Yu Z, Shi H, Yang D, Yu M (2019) MCU Up-regulation contributes to myocardial ischemiareperfusion Injury through calpain/OPA-1-mediated mitochondrial fusion/mitophagy Inhibition. J Cell Mol Med 23:7830-7843. https://doi.org/10.1111/jcmm.14662

16. Hanif A, Edin ML, Zeldin DC, Morisseau C, Nayeem MA (2016) Deletion of soluble epoxide hydrolase enhances coronary reactive hyperemia in isolated mouse heart: role of oxylipins and PPAR $\gamma$. Am J Physiol Regul Integr Comp Physiol 311:R676-r688. https:// doi.org/10.1152/ajpregu.00237.2016

17. Hausenloy DJ, Chilian W, Crea F, Davidson SM, Ferdinandy P, Garcia-Dorado D, van Royen N, Schulz R, Heusch G (2019) The coronary circulation in acute myocardial ischaemia/reperfusion injury: a target for cardioprotection. Cardiovasc Res 115:11431155. https://doi.org/10.1093/cvr/cvy286

18. Heusch G (2019) Coronary microvascular obstruction: the new frontier in cardioprotection. Basic Res Cardiol 114:45. https:// doi.org/10.1007/s00395-019-0756-8

19. Heusch G (2020) Myocardial ischaemia-reperfusion injury and cardioprotection in perspective. Nat Rev Cardiol 17:773-789. https://doi.org/10.1038/s41569-020-0403-y

20. Hong Q, Qi K, Feng Z, Huang Z, Cui S, Wang L, Fu B, Ding R, Yang J, Chen X, Wu D (2012) Hyperuricemia induces endothelial dysfunction via mitochondrial $\mathrm{Na}^{+} / \mathrm{Ca}^{2+}$ exchanger-mediated mitochondrial calcium overload. Cell Calcium 51:402-410. https://doi.org/10.1016/j.ceca.2012.01.003

21. Huo J, Lu S, Kwong JQ, Bround MJ, Grimes KM, Sargent MA, Brown ME, Davis ME, Bers DM, Molkentin JD (2020) MCUb induction protects the heart from postischemic remodeling. Circ Res 127:379-390. https://doi.org/10.1161/circresaha.119.316369

22. Jennings RB (2013) Historical perspective on the pathology of myocardial ischemia/reperfusion injury. Circ Res 113:428-438. https://doi.org/10.1161/circresaha.113.300987

23. Kavanagh DPJ, Lokman AB, Neag G, Colley A, Kalia N (2019) Imaging the injured beating heart intravitally and the vasculoprotection afforded by haematopoietic stem cells. Cardiovasc Res 115:1918-1932. https://doi.org/10.1093/cvr/cvz118

24. Klein A, Bayrau B, Miao Y, Gu M (2020) Isolation of endocardial and coronary endothelial cells from the ventricular free wall of the rat heart. J Vis Exp. https://doi.org/10.3791/61126

25. Kwong JQ, Davis J, Baines CP, Sargent MA, Karch J, Wang X, Huang T, Molkentin JD (2014) Genetic deletion of the mitochondrial phosphate carrier desensitizes the mitochondrial permeability transition pore and causes cardiomyopathy. Cell Death Differ 21:1209-1217. https://doi.org/10.1038/cdd.2014. 36

26. Lambert JP, Luongo TS, Tomar D, Jadiya P, Gao E, Zhang X, Lucchese AM, Kolmetzky DW, Shah NS, Elrod JW (2019) MCUB regulates the molecular composition of the mitochondrial calcium uniporter channel to limit mitochondrial calcium overload during stress. Circulation 140:1720-1733. https://doi.org/10.1161/circu lationaha.118.037968

27. Li C, Ma Q, Toan S, Wang J, Zhou H, Liang J (2020) SERCA overexpression reduces reperfusion-mediated cardiac microvascular damage through inhibition of the calcium/MCU/mPTP/ necroptosis signaling pathways. Redox Biol 36:101659. https:// doi.org/10.1016/j.redox.2020.101659

28. Li X, Wang M, Li S, Chen Y, Wang M, Wu Z, Sun X, Yao L, Dong H, Song Y, Xu Y (2021) HIF-1-induced mitochondrial ribosome protein L52: a mechanism for breast cancer cellular adaptation and metastatic initiation in response to hypoxia. Theranostics 11:7337-7359. https://doi.org/10.7150/thno.57804

29. Liu Y, Hu Y, Xiong J, Zeng X (2021) Overexpression of activating transcription factor 3 alleviates cardiac microvascular ischemia/ reperfusion injury in rats. Front Pharmacol 12:598959. https://doi. org/10.3389/fphar.2021.598959

30. Long L, Yu Z, Chen S, Wu J, Liu Y, Peng J, Qu H, Fu C (2021) Pretreatment of Huoxue Jiedu formula ameliorates myocardial ischaemia/reperfusion injury by decreasing autophagy via activation of the PI3K/AKT/mTOR pathway. Front Pharmacol 12:608790. https://doi.org/10.3389/fphar.2021.608790

31. Maes H, Kuchnio A, Peric A, Moens S, Nys K, De Bock K, Quaegebeur A, Schoors S, Georgiadou M, Wouters J, Vinckier S, Vankelecom H, Garmyn M, Vion AC, Radtke F, Boulanger C, Gerhardt H, Dejana E, Dewerchin M, Ghesquière B, Annaert W, Agostinis P, Carmeliet P (2014) Tumor vessel normalization by chloroquine independent of autophagy. Cancer Cell 26:190-206. https://doi.org/10.1016/j.ccr.2014.06.025

32. Martin C, Schulz R, Post H, Boengler K, Kelm M, Kleinbongard P, Gres P, Skyschally A, Konietzka I, Heusch G (2007) Microdialysis-based analysis of interstitial NO in situ: NO synthase-independent NO formation during myocardial ischemia. Cardiovasc Res 74:46-55. https://doi.org/10.1016/j.cardiores.2006.12.020

33. Martin J, Maurhofer O, Bellance N, Benard G, Graber F, Hahn D, Galinier A, Hora C, Gupta A, Ferrand G, Hoppeler H, Rossignol R, Dufour JF, St-Pierre MV (2013) Disruption of the histidine triad nucleotide-binding hint 2 gene in mice affects glycemic control and mitochondrial function. Hepatology 57:2037-2048. https://doi.org/10.1002/hep.26060

34. Milsom AB, Fernandez BO, Garcia-Saura MF, Rodriguez J, Feelisch M (2012) Contributions of nitric oxide synthases, dietary nitrite/nitrate, and other sources to the formation of NO signaling products. Antioxid Redox Signal 17:422-432. https://doi.org/10. 1089/ars.2011.4156 
35. Ndiaye D, Collado-Hilly M, Martin J, Prigent S, Dufour JF, Combettes L, Dupont G (2013) Characterization of the effect of the mitochondrial protein Hint 2 on intracellular $\mathrm{Ca}(2+)$ dynamics. Biophys J 105:1268-1275. https://doi.org/10.1016/j.bpj.2013.06. 048

36. Orrenius S, Gogvadze V, Zhivotovsky B (2015) Calcium and mitochondria in the regulation of cell death. Biochem Biophys Res Commun 460:72-81. https://doi.org/10.1016/j.bbrc.2015.01. 137

37. Pan M, Han Y, Basu A, Dai A, Si R, Willson C, Balistrieri A, Scott BT, Makino A (2018) Overexpression of hexokinase 2 reduces mitochondrial calcium overload in coronary endothelial cells of type 2 diabetic mice. Am J Physiol Cell Physiol 314:C732-c740. https://doi.org/10.1152/ajpcell.00350.2017

38. Patron M, Checchetto V, Raffaello A, Teardo E, Vecellio Reane D, Mantoan M, Granatiero V, Szabò I, De Stefani D, Rizzuto R (2014) MICU1 and MICU2 finely tune the mitochondrial Ca2+ uniporter by exerting opposite effects on MCU activity. Mol Cell 53:726-737. https://doi.org/10.1016/j.molcel.2014.01.013

39. Picca A, Mankowski RT, Burman JL, Donisi L, Kim JS, Marzetti E, Leeuwenburgh C (2018) Mitochondrial quality control mechanisms as molecular targets in cardiac ageing. Nat Rev Cardiol 15:543-554. https://doi.org/10.1038/s41569-018-0059-z

40. Pittas K, Vrachatis DA, Angelidis C, Tsoucala S, Giannopoulos G, Deftereos S (2018) The role of calcium handling mechanisms in reperfusion injury. Curr Pharm Des 24:4077-4089. https://doi. org/10.2174/1381612825666181120155953

41. Rasola A, Bernardi P (2011) Mitochondrial permeability transition in $\mathrm{Ca}(2+)$-dependent apoptosis and necrosis. Cell Calcium 50:222-233. https://doi.org/10.1016/j.ceca.2011.04.007

42. Robertson RT, Levine ST, Haynes SM, Gutierrez P, Baratta JL, Tan Z, Longmuir KJ (2015) Use of labeled tomato lectin for imaging vasculature structures. Histochem Cell Biol 143:225-234. https://doi.org/10.1007/s00418-014-1301-3

43. Seidlmayer LK, Juettner VV, Kettlewell S, Pavlov EV, Blatter LA, Dedkova EN (2015) Distinct mPTP activation mechanisms in ischaemia-reperfusion: contributions of $\mathrm{Ca} 2+, \mathrm{ROS}, \mathrm{pH}$, and inorganic polyphosphate. Cardiovasc Res 106:237-248. https:// doi.org/10.1093/cvr/cvv097
44. Skyschally A, Amanakis G, Neuhäuser M, Kleinbongard P, Heusch G (2017) Impact of electrical defibrillation on infarct size and no-reflow in pigs subjected to myocardial ischemia-reperfusion without and with ischemic conditioning. Am J Physiol Heart Circ Physiol 313:H871-h878. https://doi.org/10.1152/ajpheart.00293. 2017

45. Tan Y, Mui D, Toan S, Zhu P, Li R, Zhou H (2020) SERCA overexpression improves mitochondrial quality control and attenuates cardiac microvascular ischemia-reperfusion injury. Mol Ther Nucleic Acids 22:696-707. https://doi.org/10.1016/j.omtn.2020. 09.013

46. Tanwar J, Singh JB, Motiani RK (2020) Molecular machinery regulating mitochondrial calcium levels: the nuts and bolts of mitochondrial calcium dynamics. Mitochondrion 57:9-22. https:// doi.org/10.1016/j.mito.2020.12.001

47. Tian F, Zhang Y (2021) Overexpression of SERCA2a alleviates cardiac microvascular ischemic injury by suppressing Mfn2-mediated ER/mitochondrial calcium tethering. Front Cell Dev Biol 9:636553. https://doi.org/10.3389/fcell.2021.636553

48. Wang J, Toan S, Zhou H (2020) New insights into the role of mitochondria in cardiac microvascular ischemia/reperfusion injury. Angiogenesis 23:299-314. https://doi.org/10.1007/ s10456-020-09720-2

49. Wu L, Tan JL, Chen ZY, Huang G (2019) Cardioprotection of post-ischemic moderate ROS against ischemia/reperfusion via STAT3-induced the inhibition of MCU opening. Basic Res Cardiol 114:39. https://doi.org/10.1007/s00395-019-0747-9

50. Xue Q, Pei H, Liu Q, Zhao M, Sun J, Gao E, Ma X, Tao L (2017) MICU1 protects against myocardial ischemia/reperfusion injury and its control by the importer receptor Tom70. Cell Death Dis 8:e2923. https://doi.org/10.1038/cddis.2017.280

51. Zhou H, Shi C, Hu S, Zhu H, Ren J, Chen Y (2018) BI1 is associated with microvascular protection in cardiac ischemia reperfusion injury via repressing Syk-Nox2-Drp1-mitochondrial fission pathways. Angiogenesis 21:599-615. https://doi.org/10.1007/ s10456-018-9611-z

\section{Authors and Affiliations}

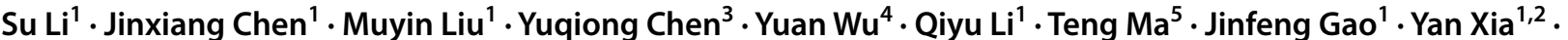

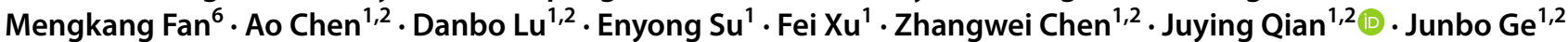

1 Department of Cardiology, Shanghai Institute of Cardiovascular Diseases, Zhongshan Hospital, Fudan University, 180 Fenglin Road, Shanghai 200032, China

2 National Clinical Research Center for Interventional Medicine, Shanghai, China

3 Department of Cardiology, The Affiliated Suzhou Hospital of Nanjing Medical University, Suzhou Municipal Hospital, Gusu School, Nanjing Medical University, Suzhou, China
4 Department of Infectious Diseases, Zhongshan Hospital, Fudan University, Shanghai, China

5 Department of Thoracic Surgery, Zhongshan Hospital, Fudan University, Shanghai, China

6 Department of Cardiology, Affiliated Hospital of Nantong University, Xisi Road 20, Nantong, Jiangsu, China 